\title{
Ambiguous representations as fuzzy relations between sets
}

\author{
Oleh Nykyforchyn ${ }^{a, *}$, Dušan Repovš ${ }^{b}$ \\ ${ }^{a}$ Vasyl' Stefanyk Precarpathian National University, Shevchenka 57, Ivano-Frankivsk, \\ 76025, Ukraine \\ ${ }^{b}$ Faculty of Mathematics and Physics and Faculty of Education, University of Ljubljana, \\ Jadranska 19, Ljubljana, 1000, Slovenia
}

\begin{abstract}
Crisp and $L$-fuzzy ambiguous representations of closed subsets of one space by closed subsets of another space are introduced. It is shown that, for each pair of compact Hausdorff spaces, the set of (crisp or $L$-fuzzy) ambiguous representations is a lattice and a compact Hausdorff Lawson upper semilattice. The categories of ambiguous and $L$-ambiguous representations are defined and investigated.
\end{abstract}

Keywords: fuzzy relation, category theory, compactum, non-additive measure, Lawson lattice.

2010 MSC: 18B10, 18B30, 54B20, 94D05

\section{Introduction}

The necessity of modeling various kinds of uncertainty, imprecision and incompleteness of information has resulted in a variety of theories which in most cases either can be reduced to two main ideas - fuzziness and roughness, or they combine the two in different ways.

A set is said to be fuzzy if, for an arbitrary element, its membership can be not only completely true or completely false, but also intermediate membership grades can occur. This level of membership can be expressed as a number in the range $[0,1]$ (classical fuzzy sets $[23]$ ), as a subinterval of $[0,1]$ (interval based fuzzy sets [5]), as a pair of two numbers with the sum $\leqslant 1$ that indicate our confidence in its membership and non-membership (vague sets, [4]), as a mapping from $[0,1]$ to $[0,1]$ (type 2 fuzzy sets, 24] ), as an element of a lattice

\footnotetext{
This research was supported by the Slovenian Research Agency grants P1-0292-0101, J1-2057-0101 and BI-UA/09-10-002, and the Ministry of Science and Education of Ukraine project M/95-2009.

Email addresses: oleh.nyk@gmail.com (Oleh Nykyforchyn), dusan.repovs@guest.arnes.si (Dušan Repovš)
} 
( $L$-fuzzy sets, [6]), etc. A membership grade of $x$ in $F$ can be interpreted in different ways [2], e.g. as proximity of $x$ to "prototype elements" of $F$, as plausibility, certainty or truth degree of " $x$ is in $F$ ", as ease ("cost") of making $x$ to "fit" into $F$ etc. We shall neither discuss nor compare different semantics of fuzzy sets, but accept a convention, which is compatible with all of them: "the more" a membership degree is, "the better" a respective element fits into a considered class of objects. Hence in the sequel "truth value" of a sentence can be understood not necessarily in the strict sense of multivalued logic, but also as degree of acceptability or certainty etc.

A set is said to be rough if it is contained in a universe where one sometimes cannot distinguish one element from another, which is formalized via partitions or equivalence relations [19]. In a rough set some elements are definitely contained, some are decidedly not members, whereas for some elements the answer is uncertain.

Nevertheless, these concepts do not completely cover uncertainty and imprecision in the description of sets. The existing tools consider it to be a derivative of uncertainty and imprecision in description/recognition/membership of individual elements [11, 12], which is insufficient. Let us imagine taking a digital photo of a text when the camera is subject to random small shifts. Then the image of a character is not uniquely determined and in order to recognize it, we cannot compare a region of a photo with a pattern on the per-pixel basis. Moreover, if all pixels of the candidate image are obtained from the pattern by a shift of 1 pixel to the left, the result is much more acceptable than if all pixels of the pattern are moved by 1 pixel in random directions, although the two possibilities are equivalent from the "elementwise" point of view.

Even though fuzzy mathematics deals with sets, e.g. when fuzzy variants of subsetness, similarity and distance between sets are investigated 9], mostly (with rare exceptions 11]) relations between subsets of the same universe are considered. We believe that a fundamental distinction between an object and its observable image, e.g. between a $3-\mathrm{D}$ body and its 2-D photo, has to be reflected in an adequate theory.

In is also important that fuzzy/rough theoretical investigations consider continuity and other topological properties of the procedures suggested to process uncertain and imprecise data (probably with only finite sets in mind). Ignoring this is rather risky because small inaccuracy can cause incorrect conclusions.

In this paper we propose a notion of a (fuzzy) ambiguous representation of subsets of one universe by subsets of another one. Sets under consideration are closed subsets of compact Hausdorff spaces, which in most cases is sufficient for applications, e.g. all closed bounded sets of $\mathbb{R}^{n}$ fit into this case. Recall also that each finite set can be regarded as a compact Hausdorff space with the discrete topology. Hence, if the reader wants to quickly gain an idea about the introduced objects, he/she can apply the following to finite sets only (and to all their subsets) and skip all topological issues. On the other hand, although it is desirable to extend our results e.g. to Tychonoff spaces or complete metric spaces (and we will do this in the future), this extension raises many complications, cf. [18] on a similar problem for inclusion hyperspaces and capacities, which are 
"building blocks" for ambiguous representations.

All compact Hausdorff spaces and (fuzzy) ambiguous representations are arranged into categories, thus allowing one to compose representations and (in some cases) to find representations that are inverse to a given one (in a special sense). It is shown that the set of "good" (fuzzy) ambiguous representations between fixed compact Hausdorff spaces is a lattice and a compact Hausdorff space as well.

The paper is organized as follows. First, all necessary definitions and facts (or references to sources) are provided in Section 1. This is followed by a strict mathematical exposition of (crisp) ambiguous representations in Section 2 and $L$-fuzzy ambiguous representations in Section 3. In Section 4 we discuss possible interpretations and applications.

\section{Preliminaries}

In the sequel a binary (ternary) relation means an arbitrary subset of the product of two (resp. three) sets. If these sets are topological spaces, we call a relation closed if it is a closed set in the product topology. For a binary relation $R \subset X \times Y$ and elements $a \in X$ and $b \in Y$ we denote $a R=\{y \in Y \mid(a, y) \in R\}$, $R b=\{x \in X \mid(x, b) \in R\}$.

For relations $R \subset X \times Y$ and $S \subset Y \times Z$, the composition of $R$ and $S$ is defined in the usual way, i.e. as

$$
\{(x, z) \in X \times Z \mid \text { there is } y \in Y \text { such that }(x, y) \in R,(y, z) \in S\} .
$$

The obtained relation is often denoted by $R \circ S$ (cf. e.g. 22]), but this contradicts to the notation for compositions of mappings. If $R \subset X \times Y$ is such that, for each $x \in X$, there is a unique $y \in Y$ such that $(x, y) \in R$, then $R$ is a mapping $X \rightarrow Y$, and the mentioned $y$ is regarded as the value $R(x)$. If $S \subset Y \times Z$ is also a mapping $Y \rightarrow Z$, then the composition of $R: X \rightarrow Y$ and $S: Y \rightarrow Z$ is a mapping $X \rightarrow Z$, which is usually denoted by $S \circ R$. To avoid confusion, we denote the composition of relations $R \subset X \times Y$ and $S \subset Y \times Z$ by $R \odot S$ (or by other similar symbols with extra circles), hence $R \odot S=S \circ R$ for mappings $R, S$.

Let $L$ be a complete distributive lattice with a bottom element 0 and a top element 1 . An $L$-fuzzy set $F$ in a universe $X$ is a mapping $F: X \rightarrow L$, with $F(x)$ being interpreted as the truth degree of the fact $x \in F$. Similarly, an $L$ fuzzy binary relation $R$ between elements of universes $X$ and $Y$ is a mapping $R: X \times Y \rightarrow L$, cf. [22]; we use either $x R y$ or $R(x, y)$ to denote the truth degree of the sentence " $x$ and $y$ are related by $R$ ". If $F(x)$ (or $R(x, y))$ takes only values 0 and 1 , then the respective set (or the relation) is called crisp and is identified with the (usual) set $\{x \in X \mid A(x)=1\}$ (resp. with the binary relation $\{(x, y) \in X \times Y \mid R(x, y)=1\})$.

For an $L$-fuzzy set $F: X \rightarrow L$ and $\alpha \in L$, the (strong) $\alpha$-cut [21] of $F$ is 
the set $F_{\alpha}=\{x \in X \mid F(x) \geqslant \alpha\}$, which is identified with the crisp set

$$
F_{\alpha}(x)=\left\{\begin{array}{l}
1, F(x) \geqslant \alpha, \\
0, F(x) \ngtr \alpha,
\end{array} \quad x \in X .\right.
$$

Similarly, for an $L$-fuzzy binary relation $R: X \times Y \rightarrow L$ and $\alpha \in L$, the $\alpha$-cut of $R[22]$ is defined as the crisp relation:

$$
R_{\alpha}(x, y)=\left\{\begin{array}{l}
1, R(x, y) \geqslant \alpha, \\
0, R(x, y) \ngtr \alpha,
\end{array} \quad x \in X, y \in Y .\right.
$$

By the above, from now on we identify $R_{\alpha}$ with the binary relation $\{(x, y) \in$ $X \times Y \mid R(x, y) \geqslant \alpha\}$.

It is obvious that the family $\left(F_{\alpha}\right)_{\alpha \in L}$ of $\alpha$-cuts (so called $L$-flou set [16]) determines an $L$-fuzzy set $F: X \rightarrow L$ completely, as well as the family $\left(R_{\alpha}\right)_{\alpha \in L}$ completely determines an $L$-relation $R: X \times Y \rightarrow L$. It is natural to "collect" these families into single subsets of $X \times L$ and $X \times Y \times L$, respectively. Hence we identify each $L$-fuzzy subset of $X$ and each $L$-relation between elements of $X$ and $Y$ with their subgraphs (or hypographs)

$$
\operatorname{sub} F=\{(x, \alpha) \in X \times L \mid \alpha \leqslant F(x)\}
$$

and

$$
\operatorname{sub} R=\{(x, y, \alpha) \in X \times Y \times L \mid \alpha \leqslant R(x, y)\}
$$

respectively.

A triple $(x, y, \alpha)$ is in $\operatorname{sub} R$ if and only if the truth degree of the sentence " $x, y$ are related by $R$ " is at least $\alpha$. For a set $S \subset X \times Y \times L$ to be a subgraph of an $L$-relation, necessary and sufficient conditions are:

(1) $S \supset X \times Y \times\{0\}$;

(2) for $x \in X, y \in Y$, and $A \subset L$ such that $(x, y, \alpha) \in S$ for all $\alpha \in A$, the triple $(x, y, \sup A)$ is also in $S$.

An obvious similar condition is valid also for subgraphs of $L$-fuzzy sets.

To define compositions of $L$-fuzzy relations, we follow [22, Section 3.3] and require that $L$ is a complete lattice, an operation $*: L \times L \rightarrow L$ is associative, commutative, infinitely distributive w.r.t. " $\mathrm{V}$ " in the both arguments, and 1 is a neutral element for "*" (i.e. $(L, *, 0,1)$ is a commutative lattice-ordered semigroup in the terminology of [22]). Then, for fuzzy relations $R: X \times Y \rightarrow L$ and $S: Y \times Z \rightarrow L$, the composition $R \odot S: X \times Z \rightarrow L$ is defined by the formula

$$
R \underset{*}{\odot} Q(x, z)=\sup \{R(x, y) * R(y, z) \mid y \in Y\}, x \in X, z \in Z .
$$

Therefore

$$
\begin{aligned}
\operatorname{sub}(R \underset{*}{\odot} Q) & =\{(x, z, \alpha) \in X \times Z \times L \mid \alpha \leqslant \sup \{\beta * \gamma \mid \\
& \text { there is } y \in Y \text { such that }(x, y, \beta) \in \operatorname{sub} R,(y, z, \gamma) \in \operatorname{sub} Q\}\} .
\end{aligned}
$$


¿From now on we shall often treat $L$-fuzzy sets and $L$-relations as subgraphs and write $F$ and $R$ instead of $\operatorname{sub} F$ and $\operatorname{sub} R$. The latter formula will be considered as the definition of composition.

We write $A \underset{\text { op }}{\subset} X$ (or $A \underset{\text { cl }}{\subset} X$ ) if $A$ is an open (resp. closed) set in a topological space $X$. A compactum is a (not necessarily metrizable) compact Hausdorff space. The category of compacta $\mathcal{C}$ omp consists of all compacta and continuous mappings between them (cf. 13] for definitions of a category and a functor). For a compactum $X$, its hyperspace $\exp X$ consists of all non-empty closed subsets of $X$. We will use the (de-facto) standard Vietoris topology [14] on $\exp X$ with a base that consists of all the sets of the form

$$
\left\langle U_{1}, \ldots, U_{n}\right\rangle=\left\{F \in \exp X \mid F \subset U_{1} \cup \cdots \cup U_{n}, F \cap U_{i} \neq \varnothing, i=1, \ldots, n\right\},
$$

with $n \in \mathbb{N}, U_{1}, \ldots, U_{n} \underset{\text { op }}{\subset} X$. The space $\exp X$ is a compactum as well, hence we can write $\exp ^{2} X=\exp (\exp X)$ etc. The Vietoris topology is the least upper bound of the upper topology with a subbase $\{\langle U\rangle \mid U \underset{\text { op }}{\subset} X\}$, and the lower topology with a subbase $\{\langle X, U\rangle \mid U \underset{\text { op }}{\subset} X\}$. A continuous mapping into $\exp X$ with the upper (lower) topology is called upper (resp. lower) semicontinuous.

If a mapping $f: X \rightarrow Y$ of compacta is continuous, then the mapping $\exp f: \exp X \rightarrow \exp Y, \exp f(F)=\{f(x) \mid x \in F\}$ for all $F \in \exp X$, is well defined and continuous. Thus the hyperspace functor $\exp$ in $\mathcal{C}$ omp is obtained.

A closed non-empty subset $\mathcal{A} \subset \exp X$ is called an inclusion hyperspace [15] if for $A, B \in \exp X$, the inclusion $B \supset A \in \mathcal{A}$ implies $B \in \mathcal{A}$. The set $G X$ of all inclusion hyperspaces is closed in $\exp ^{2} X$, therefore with the induced topology it is a compactum. This topology is determined with a subbase that consists of all the sets of the form

$$
U^{+}=\{\mathcal{A} \in G X \mid \text { there is } A \in \mathcal{A} \text { such that } A \subset U\}
$$

and

$$
U^{-}=\{\mathcal{A} \in G X \mid A \cap U \neq \varnothing \text { for all } A \in \mathcal{A}\},
$$

for all open $U \subset X$.

For any subset $\mathcal{A} \subset \exp X$ its traversal $\mathcal{A}^{\perp}=\{B \in \exp X \mid B \cap A \neq$ $\varnothing$ for all $A \in \mathcal{A}\}$ is an inclusion hyperspace, and the correspondence $\mathcal{A} \mapsto \mathcal{A}^{\perp}$ is continuous and antitone (with respect to inclusion). If $\mathcal{A} \subset \exp X$ contains all closed supersets of its elements, then $\left(\mathcal{A}^{\perp}\right)^{\perp}=\mathrm{Cl} \mathcal{A}$, hence $\left(\mathcal{A}^{\perp}\right)^{\perp}=\mathcal{A}$ if and only if $\mathcal{A} \in G X$.

A topological upper (lower) semilattice is called Lawson [10] if at each point it possesses a local base consisting of upper (resp. lower) subsemilattices. If $L$ is compact and Hausdorff, then this implies that for each $F \underset{\mathrm{cl}}{\subset} L, F \neq \varnothing$, the least upper (resp. greatest lower) bound of $F$ exists and it continuously depends on $F$. A Lawson lattice is a distributive topological lattice that is both an upper and a lower Lawson semilattice. In the sequel all topological (semi)lattices will be considered Hausdorff. The bottom and the top elements of a poset (if they 
exist) are denoted by 0 and 1 , respectively. By $\vee$ and $\wedge$ we denote resp. pairwise joins and meets. For a subset $A$ of a poset $L$, we denote:

$A \downarrow=\{\beta \in L \mid \beta \leqslant \alpha$ for some $\alpha \in A\}, \quad A \uparrow=\{\beta \in L \mid \alpha \leqslant \beta$ for some $\alpha \in A\}$.

For elements $\alpha, \beta$ of a poset $L$, we write $\alpha \ll \beta$ and say that $\alpha$ is way below $\beta$ if, for each directed set $D \subset L$ such that $\beta \leqslant \sup D$, there is an element $\gamma \in D$ such that $\alpha \leqslant \gamma$. If $L$ is a compact Lawson lattice, then this is equivalent to $\beta \in \operatorname{Int}(\{\alpha\} \uparrow)$, hence to the existence of a neighborhood $O_{\beta} \ni \beta$ such that $\alpha \leqslant \inf O_{\beta}$. The following statement is immediate:

Lemma 1.1. Let $*: L \times L \rightarrow L$ be a continuous operation that is monotone and satisfies infinite distributive laws w.r.t. inf in the both arguments. For $\alpha, \beta, \gamma \in L$, if $\gamma \ll \alpha * \beta$, then $\gamma \leqslant \alpha^{\prime} * \beta^{\prime}$ for some $\alpha^{\prime}, \beta^{\prime} \in L$ such that $\alpha^{\prime} \ll \alpha$, $\beta^{\prime} \ll \beta$.

For a compact Lawson lattice $L$ and a compactum $X$, a function $c$ : $\exp X \cup$ $\{\varnothing\} \rightarrow L$ is called an L-valued capacity [17] (or L-fuzzy measure) on a compactum $X$ if the following holds:

1. $c(\varnothing)=0, c(X)=1$;

2. for each closed subsets $F, G$ in $X$ the inclusion $F \subset G$ implies $c(F) \leqslant c(G)$ (monotonicity); and

3. if $F \subset X$ is closed and $c(F)$ lies in a neighborhood $V \subset L$, then there exists an open subset $U \supset F$ such that $c(G) \in V \downarrow$ for any closed $G \subset X$ satisfying $G \subset U$ (upper semicontinuity).

Denote by $M_{L} X$ the set of all $L$-valued capacities on a compactum $X$. We define a topology on $M_{L} X$ by a subbase that consists of all sets of the form

$$
\begin{gathered}
O_{+}(U, V)=\left\{c \in M_{L} X \mid \text { there is } F \underset{\mathrm{cl}}{\subset} U \text { such that } c(F) \geqslant \alpha \text { for some } \alpha \in V\right\}= \\
=\left\{c \in M_{L} X \mid \text { there is } F \underset{\mathrm{cl}}{\subset} U, c(F) \in V \uparrow\right\},
\end{gathered}
$$

where $U \underset{\text { op }}{\subset} X, V \underset{\text { op }}{\subset} L$, and

$O_{-}(F, V)=\left\{c \in M_{L} X \mid c(F) \leqslant \alpha\right.$ for some $\left.\alpha \in V\right\}=\left\{c \in M_{L} X \mid c(F) \in V \downarrow\right\}$,

where $F \underset{\text { cl }}{\subset} X, V \underset{\text { op }}{\subset} L$.

It was proved in [17] that the defined topology on $M_{L} X$ is compact Hausdorff. If we take a subbase that consists only of all elements of the first (second) form, we obtain the upper (resp. lower) topology on $M_{L} X$. Upper (lower) semicontinuous functions into $M_{L} X$ are defined in the obvious way.

The subgraph [17] (or hypograph) of a capacity $c \in M_{L} X$ is a set $\operatorname{sub} c=$ $\{(F, \alpha) \mid F \in \exp X, \alpha \in L, \alpha \leqslant c(F)\} \subset \exp X \times L$.

Lemma 1.2. [17] Let $X$ be a compactum, and $L$ a compact Hausdorff upper semilattice that contains the greatest element. A subset $\mathcal{F} \subset \exp X \times L$ is the 
subgraph of an L-valued capacity if and only if for all closed nonempty subsets $F, G$ of $X$ and all $\alpha, \beta \in L$ the following conditions are satisfied:

1) if $(F, \alpha) \in \mathcal{F}, \alpha \geqslant \beta$, then $(G, \beta) \in \mathcal{F}$;

2) if $(F, \alpha),(G, \beta) \in \mathcal{F}$, then $(F \cup G, \alpha \vee \beta) \in S$;

3) $\mathcal{F} \supset \exp X \times\{0\} \cup\{X\} \times L$; and

4) $\mathcal{F}$ is closed.

If these conditions hold, then the capacity $c$ is unique (and we denote it $c_{\mathcal{F}}$ ).

It was also proved in [17] that the mapping sub : $M_{L} X \rightarrow \exp (\exp X \times L)$ is an embedding.

\section{Crisp ambiguous representations}

We need several technical results.

Lemma 2.1. Let $X$ be a compactum, and let a subset $G \subset \exp X$ be such that $A \subset A^{\prime} \underset{\mathrm{cl}}{\subset} X, A \in G$ implies $A^{\prime} \in G$. Then $G$ is closed if and only if, for each filtered collection $\mathcal{F}$ of elements of $G$, we have $\bigcap \mathcal{F} \in G$.

Proof. Necessity. We can regard the aforementioned $\mathcal{F}$ as a net that converges to $\bigcap \mathcal{F}$, hence, for a closed $G$, the inclusion $\mathcal{F} \subset G$ implies $\bigcap \mathcal{F} \in G$.

Sufficiency. Let $A \in \exp X$ be a point of closure of $G$, then for all $U \underset{\text { op }}{\subset} X$ such that $A \subset U$ there is $A^{\prime} \in \exp X$ such that $A^{\prime} \in G$, and $A^{\prime} \subset U$. This implies that $\mathrm{Cl} U \in G$ for all open neighborhoods $U \supset A$. The closures of these neighborhoods form a filtered collection with the intersection $A$, hence, by assumption, $A \in G$.

Remark. A non-empty $G \subset \exp X$ that satisfies the conditions of the previous lemma is precisely an inclusion hyperspace.

Lemma 2.2. Let $X$ be a compactum, $L$ a compact Lawson upper semilattice and let a subset $R \subset \exp X \times \exp Y$ be such that, for $A, A^{\prime} \in \exp X, \alpha, \alpha^{\prime} \in L$, $A^{\prime} \supset A, \alpha^{\prime} \leqslant \alpha$, if $(A, \alpha) \in R$, then $\left(A^{\prime}, \alpha^{\prime}\right) \in R$. Then $R$ is closed if and only if the following two conditions hold:

1) for all $\alpha \in L$ and each filtered collection $\mathcal{A}$ of elements of $\exp X$ such that $\mathcal{A} \times\{\alpha\} \subset R$, we have $(\cap \mathcal{A}, \alpha) \in R$; and

2) for all $A \in \exp X$ the set of all $\alpha \in L$ such that $(A, \alpha) \in R$ is closed.

Proof. Necessity of 1) is due to the previous lemma, and is obvious for 2).

Sufficiency. Suppose that 1), 2) hold, and let $(A, \alpha) \in \exp X \times L$ be a point of the closure of $R$. For any closed neighborhood $V \supset B$ and any open neighborhood $O_{\alpha} \ni \alpha$ in $L$, there are $A^{\prime} \in \exp X, \alpha^{\prime} \in L$ such that $A^{\prime} \subset V$, $\alpha^{\prime} \in O_{\alpha},\left(A^{\prime}, \alpha^{\prime}\right) \in R$, hence $\left(V, \alpha^{\prime}\right) \in R$. Therefore $\alpha$ is a point of the closure of the set of all $\alpha^{\prime} \in L$ such that $\left(V, \alpha^{\prime}\right) \in R$, and, by 2$),(V, \alpha) \in R$. All closed neighborhoods $V \supset A$ form a net that converges to $A$, thus by 1 ) we obtain $(A, \alpha) \in R$. 
Now we are ready to introduce the main notion of this work.

Definition 2.3. An ambiguous representation between compacta $X$ and $Y$ is a subset $R \subset \exp X \times \exp Y$ such that:

a) if $A, A^{\prime} \in \exp X, B, B^{\prime} \in \exp Y, A^{\prime} \subset A, B \subset B^{\prime},(A, B) \in R$, then $\left(A^{\prime}, B^{\prime}\right) \in R$

b) $(A, Y) \in R$ for all $A \in \exp X$; and

c) for all $A \in \exp X$ the set $A R=\{B \in \exp Y \mid(A, B) \in R\}$ is closed in $\exp Y$.

Now we suggest a simple model example.

Example 2.4. Let $Y$ be the set of students, say, of a math department, and $X$ be the set of all possible marks at a test. Assume that, for $A \subset X$ and $B \subset Y$, $A, B \neq \varnothing$, we have $(A, B) \in R$ if it is likely that, after a test, $A$ is a subset of the marks of the students of $B$. Then $A$ in some sense represents the group $B$ of students, and it is obvious that a) holds. The property b) means that students' skills vary enough to result in a collection which contains any possible marks. Since both $X$ and $Y$ are finite, c) is immediate in this case, but in general it means that, if $A$ can represent sets, which are arbitrarily close to a particular $B$, then $A$ is an appropriate representation for this $B$.

In the last section there is a more extensive discussion of motivation for such a definition. The authors experienced difficulties in choosing a term for the investigated relations, in particular because most "good words" like "fuzzy", "rough", "vague" etc have been already "occupied". We needed to express two features: one set is not necessarily a copy or an image of another one (although this is also possible), but represents it in some manner, which may vary; and there can be many valid representatives for a set, as well as a set can represent many sets, hence such a relation is ambiguous. Thus we have arrived at "ambiguous representations".

Nonetheless, the just introduced concept is not quite unrelated to rough sets.

Example 2.5. Let $\sim$ be an indiscernibility relation on a finite set $X$, i.e. it is an equivalence relation that contains all pairs of elements $x, y \in X$ such that we cannot distinguish $x$ from $y$. Then two subsets $A, B$ are indistinguishable if and only if their upper approximations $\overline{a p p r}_{\sim} A=\{x \in X \mid x \sim$ $a$ for some $a \in A\}$ and $\overline{a p p r} \sim B=\{y \in X \mid y \sim b$ for some $b \in B\}$ are equal. Let $R \subset \exp X \times \exp X$ consist from all pairs $(A, B)$ such that non-empty $A$ is indistinguishable from a subset of $B$, i.e. $\overline{a p p r}_{\sim} A \subset \overline{a p p r} \sim B$. Then $R$ is an ambiguous representation between $X$ and $X$.

In the next section the concept of ambiguous representation will also be modified in the spirit of fuzzy mathematics.

We denote the set of all ambiguous representations between $X$ and $Y$ by $\mathcal{C} \mathcal{A m b}(X, Y)$.

Definition 2.6. If $R$ is an ambiguous representation, and $(A, B) \in R$, we say that $B$ is $R$-admissible for $A$. If $C \underset{\text { cl }}{\subset} Y, C \cap B \neq \varnothing$ for all $B \in A R$, we call $C$ an $R$-unavoidable set for $A$. 
Example 2.7. Let $R \subset \exp X \times \exp X$ be the relation defined in the previous example. Then $C \subset X$ is $R$-unavoidable for $A \subset X$ if and only if the upper approximation $\overline{a_{p p r}} \sim A$ has non-empty intersection with the lower approximation $\underline{\text { appr }} \sim C=\{c \in C \mid x \in C$ for all $x \in X, x \sim c\}$.

Example 2.8. Let $C$ be the set of all students of a department that are able to obtain a highest mark. If a set $A$ contains such a mark and represents a set $B$ of students, then $B$ must contain at least one well-prepared student, hence $B \cap C \neq \varnothing$, and $C$ in unavoidable for $A$.

For an ambiguous representation $R \subset \exp X \times \exp Y$ we define the relation $R^{\smile} \subset \exp Y \times \exp X$ as follows:

$$
\begin{aligned}
R^{\smile}=\{(\tilde{B}, \tilde{A}) \in \exp Y & \times \exp X \mid \\
\forall A & \in \exp X(A \cap \tilde{A}=\varnothing \Longrightarrow \exists B \in A R B \cap \tilde{B}=\varnothing)\},
\end{aligned}
$$

i.e. $(\tilde{B}, \tilde{A}) \in R^{\smile}$ if and only if $\tilde{A}$ has non-empty intersections with all $A \in \exp X$ such that $\tilde{B}$ is $R$-unavoidable for $A$. Thus

$$
\tilde{B} R^{\smile}=\left\{A \in \exp X \mid \tilde{B} \in(A R)^{\perp}\right\}^{\perp}
$$

for all $\tilde{B} \in \exp Y$.

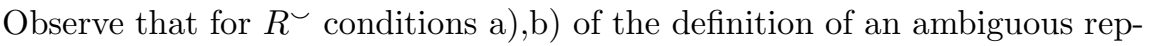
resentation between $Y$ and $X$ obviously hold. The previous formula implies c), hence $R^{\smile} \in \mathcal{C} \mathcal{A m b}(Y, X)$.

Example 2.9. Let $X$ and $Y$ be the squares $[0,1] \times[0,1]$ and $[0,1] \times[1,2]$, respectively. For $A \in \exp X, B \in \exp Y$, assume $(A, B) \in R$ if $\operatorname{pr}_{1}(A) \subset \operatorname{pr}_{1}(B)$. In other words, $A$ can represent $B$ if and only if it is covered by the "shade" of $B$ under vertical light. Then $(\bar{B}, \bar{A}) \in \exp Y \times \exp X$ is in $R^{\smile}$ if and only if $\operatorname{pr}_{1}(X \backslash \bar{A}) \subset \operatorname{pr}_{1}(Y \backslash \bar{B})$, i.e. each point outside of $\bar{A}$ is in the "shade" of a point outside of $\bar{B}$.

Proposition 2.10. If $R \subset \exp X \times \exp Y$ is an ambiguous representation, then $\left(R^{\smile}\right)^{\smile} \subset R$.

Proof. For all $A \in \exp X$ :

$$
\begin{gathered}
A\left(R^{\smile}\right)^{\smile}=\left\{\tilde{B} \in \exp Y \mid A \in\left(\tilde{B} R^{\smile}\right)^{\perp}\right\}^{\perp}= \\
\left\{\tilde{B} \in \exp Y \mid A \in\left\{A^{\prime} \in \exp X \mid \tilde{B} \in\left(A^{\prime} R\right)^{\perp}\right\}^{\perp \perp}\right\}^{\perp}= \\
\left\{\tilde{B} \in \exp Y \mid A \in \operatorname{Cl}\left\{A^{\prime} \in \exp X \mid \tilde{B} \in\left(A^{\prime} R\right)^{\perp}\right\}\right\}^{\perp}= \\
\left\{\tilde{B} \in \exp Y \mid \tilde{B} \in(V R)^{\perp} \text { for all } V \underset{\mathrm{cl}}{\subset} X, A \subset \operatorname{Int} V\right\}^{\perp} \subset \\
\left\{\tilde{B} \in \exp Y \mid \tilde{B} \in(A R)^{\perp}\right\}^{\perp}=(A R)^{\perp \perp}=A R .
\end{gathered}
$$

Corollary 2.11. For an ambiguous representation $R \subset \exp X \times \exp Y$, the equality $\left(R^{\smile}\right)^{\smile}=R$ is valid if and only if:

d) for all $(A, B) \in R$ and a closed neighborhood $V \supset B$ there is a closed neighborhood $U \supset A$ such that $(U, V) \in R$. 
Proof. ¿From the latter formula it is easy to obtain an explicit expression for $\left(R^{\smile}\right)^{\smile}$ : if $A \in \exp X$, then

$$
A\left(R^{\smile}\right)^{\smile}=\mathrm{Cl}(\bigcup\{V R \mid V \text { is a closed neighborhood of } A\}) .
$$

Definition 2.12. If $R \subset \exp X \times \exp Y$ is an ambiguous representation such that $\left(R^{\smile}\right)^{\smile}=R$, that we call $R^{\smile}$ pseudo-inverse to $R$, and $R$ is called pseudoinvertible.

Remark. It is obvious that then $R$ is pseudo-inverse to $R^{\smile}$, and $R^{\smile}$ is pseudoinvertible as well.

The set of all pseudo-invertible ambiguous representations between $X$ and $Y$ is denoted by $\mathcal{C P} \mathcal{A m b}(X, Y)$.

The composition of ambiguous representations $R \in \mathcal{C} \mathcal{A m b}(X, Y)$ and $S \in$ $\mathcal{C} \mathcal{A m b}(Y, Z)$ is defined in the usual way :

$$
\begin{aligned}
R \odot S=\{ & (A, C) \in \exp X \times \exp Z \mid \text { there is } B \in \exp Y \\
& \text { such that }(A, B) \in R,(B, C) \in S\} .
\end{aligned}
$$

It is easy, however, to find an example of two ambiguous representations (even pseudo-invertible ones) such that their composition is not an ambiguous representation. To obtain a category, we have two options: either to modify the composition law, or to restrict the class of allowed relations.

For ambiguous representations $R \subset \exp X \times \exp Y$ and $S \subset \exp Y \times \exp Z$, let a relation $R \bar{\odot} S \subset \exp X \times \exp Z$ be defined by the equality $A(R \bar{\odot} S)=$ $\mathrm{Cl}(A(R \odot S))$ for all $A \in \exp X$ (the closure is taken w.r.t. the Vietoris topology). In other words, $C \in A(R \bar{\odot} S)$ if and only if for all closed neighborhoods $V \subset C$ there is $B \in \exp Y$ such that $(A, B) \in R,(B, V) \in S$. Then $R \odot S$ is an ambiguous representation. Unfortunately, the equality $(R \bar{\odot} S) \bar{\odot} T=R \bar{\odot}(S \bar{\odot} T)$ is not valid in general, hence compacta and ambiguous representations do not form a category.

Lemma 2.13. For ambiguous representations $R \subset \exp X \times \exp Y, S \subset \exp Y \times$ $\exp Z$ the inclusion $S \smile \bar{\odot} R^{\smile} \subset(R \odot S)^{\smile}$ is valid.

The proof is straightforward.

Proposition 2.14. Let $R \subset \exp X \times \exp Y$ and $S \subset \exp Y \times \exp Z$ be pseudoinvertible ambiguous representations. Then $R \odot S \subset \exp X \times \exp Z$ is a pseudoinvertible ambiguous representation as well, and $(R \bar{\odot} S)^{\smile}=S^{\smile} \bar{\odot} R^{\smile}$.

Proof. By Lemma 2.13,

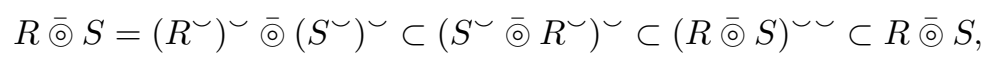

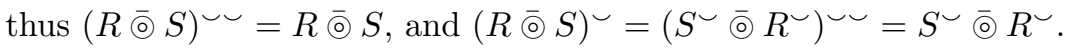


Due to Corollary 2.11 the composition law $\bar{\odot}$ is associative for pseudoinvertible ambiguous representations. Hence all compacta and pseudo-invertible ambiguous representations form a category $\mathcal{C} \mathcal{P} \mathcal{A m b}$. In this category a pseudoinvertible ambiguous representation $R \subset \exp X \times \exp Y$ is considered as an arrow $X \rightarrow Y$, and for an arrow $S: Y \rightarrow Z$, i.e. for a pseudo-invertible ambiguous representation $S \subset \exp Y \times \exp Z$, the composition $S \circ R$ is equal to $R \bar{\odot} S$. For a compactum $X$, the identity arrow $\mathbf{1}_{X}$ is equal to $\{(A, B) \in \exp X \times \exp X \mid A \subset B\}$.

Observe also that $\mathbf{1}_{X}{ }^{\smile}=\mathbf{1}_{X}$, therefore we obtain an involutive antiisomorphism $(-)^{\smile}: \mathcal{C P} \mathcal{A} \mathrm{mb}^{o p} \rightarrow \mathcal{C P} \mathcal{A} \mathrm{mb}$, which preserves objects.

To use the second variant, i.e. to construct a class of relations that is closed under composition, we add the following requirement:

e) for all $B \in \exp X$ the set $R B=\{A \in \exp X \mid(A, B) \in R\}$ is closed in $\exp X$.

Definition 2.15. A relation $R \subset \exp X \times \exp Y$ that satisfies a)-c) and e) is called a strict ambiguous representation between $X$ and $Y$.

Observe that $(\exp X, \subset)$ is a compact Lawson upper semilattice, hence we can use Lemma 2.2 to give an equivalent definition:

Definition 2.16. A subset $R \subset \exp X \times \exp Y$ is a strict ambiguous representation if $R$ is closed, contains $\exp X \times\{Y\}$, and $(A, B) \in R, A^{\prime} \in \exp X$, $B^{\prime} \in \exp Y, A^{\prime} \subset A, B^{\prime} \supset B$ imply $\left(A^{\prime}, B^{\prime}\right) \in R$.

We denote the set of all strict ambiguous representations between $X$ and $Y$ by $\mathcal{C} \mathcal{S} \mathcal{A m b}(X, Y)$. The intersection $\mathcal{C P} \mathcal{A m b}(X, Y) \cap \mathcal{C S} \mathcal{A m b}(X, Y)$ is denoted by $\mathcal{C P S} \mathcal{A m b}(X, Y)$.

Proposition 2.17. For compacta $X, Y$, a relation $R \subset \exp X \times \exp Y$ is an ambiguous representation if and only if the correspondence $A \mapsto A R$ is an antitone mapping from $\exp X$ to $G Y$. This mapping is:

- lower semicontinuous if and only if $R$ is pseudo-invertible;

- upper semicontinuous if and only if $R$ is strict;

- continuous if and only if $R$ is pseudo-invertible and strict.

The proof is straightforward and it uses Lemma 2.1.

Since the composition of compact relations is compact, we immediately obtain:

Proposition 2.18. If $R \in \mathcal{C} \mathcal{S} \mathcal{A m b}(X, Y)$ and $S \in \mathcal{C} \mathcal{S A m b}(Y, Z)$, then $R \odot S \in$ $\mathcal{C S A m b}(X, Z)$.

Remark. For a reader to observe ambiguous representations "at work", we also provide an independent proof of c) and d) (properties a), b) are obviously satisfied).

Let us observe that $A(R \odot S) \subset \exp Z$ contains supersets of all its elements and the entire space $Z$. To prove closedness, we use Lemma 2.1, assume that $\mathcal{F} \subset A(R \odot S)$ is a filtered collection, and let $C_{0}=\bigcap \mathcal{F}$. Observe that $(A, C) \in$ $R \odot S$ if and only if $A R \cap S C \neq \varnothing$. By c) $A R \subset \exp Y$ is an inclusion hyperspace, 
and by d) the collection $\{S C \mid C \in \mathcal{F}\}$ is a decreasing net of closed subsets of $\exp Y$ if $\mathcal{F}$ is ordered reverse to inclusion. Each of $\{S C \mid C \in \mathcal{F}\}$ has a nonempty intersection with $A R$, hence there is $B \in A R \cap \bigcap\{S C \mid C \in \mathcal{F}\}$. For any neighborhood $U \subset C_{0}$ there is $C \in \mathcal{F}$ such that $C \subset \mathrm{Cl} U$, therefore $(B, C) \in S$ implies $(B, \mathrm{Cl} U) \in S$. Using c) again, we obtain $C_{0} \in A(R \odot S)$, i.e. c) for $R \odot S$.

Let $(A, B) \in \exp X \times \exp Z \backslash R \odot S$, then $A R \cap S C=\varnothing$. Due to the compactness of $S C$ there are open sets $U_{1}, \ldots, U_{n} \subset Y$ such that all $B \in S C$ are contained in some $U_{i}$, and no $\mathrm{Cl} U_{i}$ is an element of $A R$. Then $A \in \mathcal{V}=$ $\exp X \backslash\left(R \mathrm{Cl} U_{1} \cup \cdots \cup R \mathrm{Cl} U_{n}\right)$, and $\mathcal{V}$ is an open neighborhood of $A$ in $\exp X$ such that $A^{\prime} \in \mathcal{V}$ is incompatible with $\left(A^{\prime}, C\right) \in R \odot S$. Thus $(R \odot S) C$ is closed.

Therefore we can define the category of compacta and strict ambiguous representations $\mathcal{C} \mathcal{S} \mathcal{A m b}$. Arrows from $X$ to $Y$ in this category are the strict ambiguous representations between $X$ and $Y$, the composition of $R: X \rightarrow Y$ and $S: Y \rightarrow Z$ is the usual composition $R \odot S$ of relations, identity arrows are of the same form as in $\mathcal{C P} \mathcal{A m b}$. For strict ambiguous representations the compositions $\odot$ and $\bar{\odot}$ coincide, hence we can consider the intersection of $\mathcal{C P} \mathcal{A m b}$ and $\mathcal{C S A m b}$, which we call the category of compacta and strict pseudo-invertible ambiguous representations and denote by $\mathcal{C P} \mathcal{S} \mathcal{A m b}$.

A drawback of introduction of e) is that the ambiguous representation $R^{\smile}$ for $R \in \mathcal{C} \mathcal{S} \mathcal{A m b}(X, Y)$ is not always strict.

Example 2.19. Let $f: X \rightarrow Y$ be a continuous mapping of compacta, and a relation $R_{f} \subset \exp X \times \exp Y$ be defined as $(A, B) \in R_{f}$ if and only if $f(A) \subset$ $B$. Then $R_{f}$ is a pseudo-invertible strict ambiguous representation, and for all $B \in \exp Y$ we have

$$
\begin{gathered}
B R_{f}^{\smile}=\left\{A \in \exp X \mid B \in\{f(A)\}^{\perp}\right\}^{\perp}= \\
\{A \in \exp X \mid f(A) \cap B \neq \varnothing\}^{\perp}=\left\{A \in \exp X \mid A \supset f^{-1}(B)\right\} .
\end{gathered}
$$

Therefore

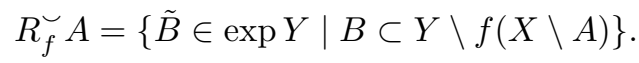

It is easy to see that the latter set is closed for all closed $A$ if and only if the mapping $f$ is open.

Definition 2.20. An ambiguous representation $R$ is called an open ambiguous representation if it is strict, pseudo-invertible and $R^{\smile}$ is a strict ambiguous representation.

Remark. Here we do not mean that $R$ is an open subset in the product.

Proposition 2.21. A relation $R \subset \exp X \times \exp Y$ that satisfies a) $-e)$ is an open ambiguous representation if and only if any of the following statements is valid:

f) for any open $U \subset X$ the set of $R$-unavoidable sets of all $A \subset U, A \in$ $\exp X$, is open in the Vietoris topology on $\exp Y$; 
f') for all $A \in \exp X, A \subset U \underset{\text { op }}{\subset} X$ and $B \in \exp Y$ such that $B \in(A R)^{\perp}$, there are open sets $V_{1}, \ldots, V_{n} \subset Y$ and a closed neighborhood $G \supset F$ in $X$ such that $G \subset U, V_{i} \cap B \neq \varnothing, i=1, \ldots, n$, and each $B^{\prime} \in G R$ contains at least one of $V_{i}$.

Proof. We need to verify only the closedness of $R \smile \tilde{A}$ for all $\tilde{A} \in \exp X$. Observe that

$$
\begin{aligned}
& R^{\smile} \tilde{A}=\left\{\tilde{B} \in \exp Y \mid \forall A \in \exp X\left(\tilde{B} \in(A R)^{\perp} \Longrightarrow A \cap \tilde{A} \neq \varnothing\right)\right\}= \\
& \{\tilde{B} \in \exp Y \mid \forall A \in \exp X \quad(A \cap \tilde{A}=\varnothing \Longrightarrow \exists B \in A R \quad B \cap \tilde{B}=\varnothing)\} .
\end{aligned}
$$

Hence

$$
\exp Y \backslash R^{\smile} \tilde{A}=\bigcup\left\{(A R)^{\perp} \mid A \in \exp X, A \subset X \backslash \tilde{A}\right\} .
$$

The latter set is the complement to the set of all $R$-unavoidable sets for all $A \underset{\text { cl }}{\subset} X, A \subset U=X \backslash \tilde{A}$. Thus f) is equivalent to the closedness of $R \smile \tilde{A}$ for all $\tilde{B} \in \exp A$.

The equivalence of $f$ ) and $f^{\prime}$ ) is a particular case of Proposition 3.14, which will be proved in the next section.

Corollary 2.22. The class of open ambiguous representations is closed under composition.

Thus we obtain the category of compacta and open ambiguous representations $\mathcal{C O} \mathcal{A m b}$ that is a subcategory of $\mathcal{C P} \mathcal{A m b}$, and the restriction of the contravariant functor $(-)^{\smile}$ to $\mathcal{C O} \mathcal{A m b}$ is also an involutive antiisomorphism, which preserves objects.

Now we consider order properties of the sets of ambiguous representations. Observe that $\mathcal{C} \mathcal{A m b}(X, Y)$ is a lattice when ordered by inclusion, i.e. $R$ precedes $S$ if $R \subset S$. For $R, S \in \mathcal{C} \mathcal{A m b}(X, Y)$ the meet of $R, S$ is equal to $R \cap S$, and their join is equal to $R \cup S$. The subsets $\mathcal{C} \mathcal{S} \mathcal{A m b}(X, Y)$ and $\mathcal{C P} \mathcal{A m b}(X, Y)$ (and therefore $\mathcal{C} \mathcal{P} \mathcal{S} \mathcal{A m b}(X, Y))$ are sublattices of $\mathcal{C} \mathcal{A} \operatorname{mb}(X, Y)$. To prove a similar fact about $\mathcal{C O} \mathcal{A m b}(X, Y)$, we need the following statement.

Proposition 2.23. If $R, S \in \mathcal{C O} \mathcal{A m b}(X, Y)$, then $R \cap S, R \cup S \in \mathcal{C O} \mathcal{A m b}(X, Y)$. Proof. Only f) has to be checked for $R \cap S, R \cup S$. It is easy to see that $C \in \exp Y$ is $R \cap S$-unavoidable for $A \in \exp X$ if and only if $C$ is either $R$-unavoidable or $S$-unavoidable. Therefore for any open $U \subset X$ the set of $R \cap S$-unavoidable sets of all $A \subset U, A \in \exp X$, is equal to the union of the sets of all $R$-unavoidable and of all $S$-unavoidable sets for all $A \subset U, A \in \exp X$, thus it is open in the Vietoris topology on $\exp Y$.

Similarly, $C \in \exp Y$ is $R \cup S$-unavoidable for $A \in \exp X$ if and only if $C$ is $R$-unavoidable and $S$-unavoidable. Let $U \underset{\text { op }}{\subset} X, A \in \exp X$ and $B \in \exp Y$ be such that $A \subset U$ and $B$ is $R \cup S$-unavoidable for $A$. By assumption there is a neighborhood $O C \ni C$ in $\exp Y$ such that each $C^{\prime} \in O C$ is $R$-unavoidable for some $A_{1} \subset U$ and $S$-unavoidable for some $A_{2} \subset U, A_{1}, A_{2} \in \exp X$. Then such $C^{\prime}$ is $R \cap S$-unavoidable for $A^{\prime}=A_{1} \cup A_{2} \subset U$, which completes the proof. 
Corollary 2.24. The set $\mathcal{C O} \mathcal{A m b}(X, Y)$ is a sublattice of $\mathcal{C P S} \mathcal{A m b}(X, Y)$.

This statement can also be derived from:

Proposition 2.25. Let $R, S \in \mathcal{C} \mathcal{A m b}(X, Y)$, then $(R \cup S)^{\smile}=R^{\smile} \cup S^{\smile},(R \cap$ $S)^{\smile}=R^{\smile} \cap S^{\smile}$.

The proof is straightforward, see also more general Proposition 3.16,

The top and the bottom elements in the posets $\mathcal{C} \mathcal{A m b}(X, Y), \mathcal{C} \mathcal{S} \mathcal{A m b}(X, Y)$, and $\mathcal{C P} \mathcal{A m b}(X, Y)$ are determined by the equalities:

$$
\top_{X, Y}=\exp X \times \exp Y, \perp_{X, Y}=\exp X \times\{Y\} .
$$

Observe that $\top_{X, Y}, \perp_{X, Y}$ are not always in $\mathcal{C O} \mathcal{A m b}(X, Y)$.

Question 2.26. For which compacta $X, Y$ there are top and bottom elements in $\mathcal{C O} \mathcal{A m b}(X, Y)$ ?

The answer is trivially positive for finite compacta.

Observe that all subsets of $\exp X \times \exp Y$ that satisfy the definition 2.16 form a closed subsemilattice of the compact Lawson upper semilattice $\exp (\exp X \times$ $\exp Y$ ), thus:

Proposition 2.27. The set $\mathcal{C} \mathcal{S} \mathcal{A m b}(X, Y)$ is a compact Lawson upper semilattice.

Observe that for non-finite $X, Y$ the lattice $\mathcal{C} \mathcal{S} \mathcal{A m b}(X, Y)$ is not topological, for meet (=intersection) is not continuous in general w.r.t. the Vietoris topology.

Question 2.28. What are topological properties of the subsets $\mathcal{C P} \mathcal{S} A \operatorname{mb}(X, Y)$, $\mathcal{C O} \mathcal{A m b}(X, Y)$ of $\mathcal{C} \mathcal{S} \mathcal{A m b}(X, Y)$ ?

For the operation $(-)^{\smile}$ is involutive and isotonic, it provides isomorphisms of lattices $\mathcal{C P} \mathcal{A m b}(X, Y) \cong \mathcal{C P} \mathcal{A m b}(Y, X)$ and $\mathcal{C O} \mathcal{A m b}(X, Y) \cong \mathcal{C O} \mathcal{A m b}(Y, X)$ for all compacta $X, Y$.

Recall that an allegory [22] is a category $\mathcal{C}$ in which:

1) for all objects $X, Y$ each set $\mathcal{C}(X, Y)$ is a lower semilattice; we denote its meet (also called intersection in this case) and order by $\wedge$ and $\prec$, respectively;

2) for all objects $X, Y$ there is a monotonic operation $(-)^{\smile}$ (anti-involution or converse operation), that takes every morphism $f: X \rightarrow Y$ to a morphism $f^{\smile}: Y \rightarrow X$, such that $\left(f^{\smile}\right)^{\smile}=f$ and $(f \circ g)^{\smile}=g^{\smile} \circ f^{\smile}$, provided that $f \circ g$ exists;

3) composition is monotone in both arguments; and

4) the modular law holds: if $f: X \rightarrow Y, g: Y \rightarrow Z, h: X \rightarrow Z$, then $g \circ f \wedge h \prec\left(g \wedge h \circ f^{\smile}\right) \circ f$.

We can see that $\mathcal{C P} \mathcal{A m b}$ and $\mathcal{C O} \mathcal{A m b}$ with the defined operation $(-)^{\smile}$ satisfy all the requirements of the definition of allegory but the last (which is not too surprising, because we generalize mappings rather than relations). Moreover, composition is distributive over meet and join in the both arguments. 


\section{3. $L$-ambiguous representations}

In the sequel $L$ will be a compact Lawson lattice, 0 and 1 the bottom and the top elements of $L$. To fit into a described in the introductory section $L$ fuzzy framework, we consider an operation $*: L \times L \rightarrow L$, which is associative, commutative, isotone in the both arguments and 1 is a neutral element for "*". We demand that $*$ be lower semicontinuous and distributive w.r.t. $\vee$ in the both arguments. Due to compactness this implies infinite distributive laws. Sometimes we shall need also the upper semicontinuity, i.e. the continuity of $*$. The simplest such "*" is the lattice meet " $\wedge "$. See also [1] for more information on such operations.

For each subset $R \subset X \times Y \times L$ (not only for a subgraph of an $L$-relation) and $\alpha \in L$, we define the $\alpha$-cut $R_{\alpha}$ as follows

$$
R_{\alpha}=\{(a, b) \in X \times Y \mid(a, b, \alpha) \in R\} .
$$

For subsets $A \subset X, B \subset Y$ we put

$$
\begin{aligned}
& A R=\{(y, \alpha) \in Y \times L \mid \text { there is } x \in A \text { such that }(x, y, \alpha) \in R\}, \\
& R B=\{(x, \alpha) \in X \times L \mid \text { there is } y \in B \text { such that }(x, y, \alpha) \in R\} .
\end{aligned}
$$

Definition 3.1. Let $X, Y$ be compacta, $L$ be a compact Lawson lattice. A subset $R \subset \exp X \times \exp Y \times L$ is called an $L$-ambiguous representation between $X$ and $Y$ if:

a) if $A, A^{\prime} \in \exp X, B, B^{\prime} \in \exp Y, \alpha, \alpha^{\prime} \in L, A^{\prime} \subset A, B \subset B^{\prime}, \alpha \geqslant \alpha^{\prime}$, then $(A, B, \alpha) \in R$ implies $\left(A^{\prime}, B^{\prime}, \alpha^{\prime}\right) \in R$;

b) if $A \in \exp X, B \in \exp Y, \alpha, \beta \in L$ are such that $(A, B, \alpha),(A, B, \beta) \in R$, then $(A, B, \alpha \vee \beta) \in R$;

c) $(A, Y, \alpha),(A, B, 0) \in R$ for all $A \in \exp X, B \in \exp Y, \alpha \in L$; and

d) for all $A \in \exp X$ the set $A R=\{(B, \alpha) \in \exp Y \times L \mid(A, B, \alpha) \in R\}$ is closed in $\exp Y \times L$.

It is equivalent to $R$ being a subgraph of an $L$-fuzzy binary relation between $\exp X$ and $\exp Y$ (denoted by the same letter $R$ for brevity) such that:

a') $R$ is antitone in the first argument;

b') $R$ is isotone and upper semicontinuous (i.e. preserves filtered infima) in the second argument;

c') $R(A, Y)=1$ for all $A \in \exp X$.

The mapping $R: \exp X \times \exp Y \rightarrow L$ is uniquely recovered by the formula $R(A, B)=\max \{\alpha \in L \mid(A, B, \alpha) \in R\}$. The value $R(A, B) \in L$ is interpreted as a degree which shows how well $A$ can represent $B$ (the more, the better). If $(A, B, \alpha) \in R$, then $A$ represents $B$ with fitness at least $\alpha$. We shall interchange the relational and the functional interpretations of $L$-ambiguous representations, whatever is more convenient in a particular case.

Example 3.2. Let $(X, d)$ be a metric compactum and $L=[0,1]$. We put

$$
R(A, B)=1-\sup \{d(a, B) \mid a \in A\} / \operatorname{diam} X, A \in \exp X, B \in \exp Y .
$$


Then $R(A, B) \geqslant \alpha$ if and only if $A$ extends beyond $B$ by no more that $\delta=$ $(1-\alpha) \operatorname{diam} X$. It means that $A$ can be obtained from a closed subset $B_{0} \subset B$ by "shifts" of its points by $\leqslant \delta$ in different (probably multiple) directions.

Example 3.3. Let $X \subset Y$ be compact subsets of $\mathbb{R}^{2}$, diam $Y=r, L=[0, r]$. For $A \in \exp Y, B \in \exp Y$, we put

$$
R(A, B)=r-\inf \left\{\|\vec{m}\| \mid \vec{m} \in \mathbb{R}^{2}, A+\vec{m} \subset B\right\},
$$

assuming $\inf \varnothing=r$. Then $R(A, B) \geqslant \alpha>0$ iff $A$ can be shifted in one direction by a distance $\leqslant r-\alpha$ to coincide with a subset of $B$.

The two latter examples implement a common idea: $R(A, B)$ shows how well a subset $A$ represents a part of an image of $B$. Of course, we can combine shifts, expansions, rotations etc, depending on which distortions of images are expected. It is also not necessary that $X$ and $Y$ be of equal dimension. Assume, e.g., that $Y$ is an area in atmosphere, and $X$ is a finite set of points on the earth surface where automatic registration devices are installed. If there is a snow cloud somewhere in $Y$, and $A \subset X$ is a (probably incomplete) set of points where snowfall is observed, then it is possible to define a function $R(A, B)$, which will estimate the likelihood that the cloud is contained in a subset $B \subset Y$. Such problems, where uncertainty, distortions and incompleteness of information combine, are the main target of the introduced $L$-fuzzy ambiguous representations.

Now we discuss how they are related to concepts used in fuzzy sets theory, in particular, in fuzzy topology. The latter theory in its different flavors [20] studies crisp or fuzzy families of fuzzy subsets of a universe. We are not going so far in "fuzzification", and only crisp or fuzzy relations between hyperspaces of ordinary closed subsets of compacta are considered, although "totally fuzzy" generalizations of ambiguous representations can also be introduced. Probably, to develop a consistent theory, these future extensions would require use of sheaf-theoretic apparatus [7, [8]. Hence, similarly to fuzzy topology in its "more fuzzy" variants, different degrees of membership of a set in a family of valid representatives for another set can occur. We see no reasons to restrict ourselves to the unit interval to express membership, and prefer Goguen's lattice-valued approach [6]. Note that we use lattice elements to describe rather quality of representations, which does not necessarily relates to probabilistic interpretation of fuzzy sets.

Of course, even this "moderate" $L$-fuzziness of our constructions inevitably leads to "graded" families similar to studied by Negoita and Ralescu [16]. Observe that our level cuts are not sets of individual points, but relations between hyperspaces.

The set of all $L$-ambiguous representations between $X$ and $Y$ is denoted by $\mathcal{C} \mathcal{A m b}_{L}(X, Y)$.

Definition 3.4. An $L$-ambiguous representation $R \subset \exp X \times \exp Y \times L$ is strict if for all $B \in \exp Y$ the set $R B=\{(A, \alpha) \in \exp X \times L \mid(A, B, \alpha) \in R\}$ is closed in $\exp X \times L$. 
We denote the set of all strict $L$-ambiguous representations between $X$ and $Y$ by $\mathcal{C} \mathcal{S} \mathcal{A m b}_{L}(X, Y)$.

By the following lemma a strict $L$-ambiguous representation $R \subset \exp X \times$ $\exp Y \times L$ is a closed subset.

Lemma 3.5. Let $X, Y$ be compacta, $L$ a compact Lawson upper semilattice, and let a subset $R \subset \exp X \times \exp Y \times L$ be such that, for $A, A^{\prime} \in \exp X$, $B, B^{\prime} \in \exp Y, \alpha, \alpha^{\prime} \in L, A^{\prime} \subset A, B \subset B^{\prime}, \alpha^{\prime} \leqslant \alpha$, if $(A, B, \alpha) \in R$, then $\left(A^{\prime}, B^{\prime}, \alpha^{\prime}\right) \in R$. Then $R$ is closed if and only if the following two conditions hold:

1) for all $A \in \exp X, \alpha \in L$ and each filtered collection $\mathcal{B}$ of elements of $\exp Y$ such that $\{A\} \times \mathcal{B} \times\{\alpha\} \subset R$, we have $(A, \cap \mathcal{B}, \alpha) \in R$; and

2) for all $B \in \exp Y$ the set of all $(A, \alpha) \in \exp X \times L$ such that $(A, B, \alpha) \in R$ is closed.

This can be derived from Lemma 2.2 by a simple observation that $\exp X \times L$ is a compact Lawson upper semilattice.

For $L$-ambiguous representations $R \subset \exp X \times \exp Y \times L, S \subset \exp Y \times$ $\exp Z \times L$ we define the composition $R \underset{*}{\odot} S$ in the following manner, which is customary for $L$-relations (cf. the introductory section):

$$
\begin{aligned}
& \underset{*}{R} S=\{(A, C, \alpha) \in \exp X \times \exp Z \times L \mid \alpha \leqslant \sup \{\beta * \gamma \mid \\
& \text { there is } B \in \exp Y \text { such that }(A, B, \beta) \in R,(B, C, \gamma) \in S\}\},
\end{aligned}
$$

or, equivalently, in the functional notation:

$$
R \underset{*}{\odot} S(A, C)=\sup \{R(A, B) * S(B, C) \mid B \in \exp Y\},
$$

for $A \in \exp X, C \in \exp Z$.

Proposition 3.6. If $*: L \times L \rightarrow L$ is continuous, $R \in \mathcal{C} \mathcal{S A m b}_{L}(X, Y)$, and $S \in \mathcal{C S} \mathcal{A m b}_{L}(Y, Z)$, then $R \underset{*}{\odot} S$ is a strict $L$-ambiguous representation.

Proof. The set

$$
\begin{aligned}
& R \underset{*}{\odot \odot S} S=\{(A, C, \alpha) \in \exp X \times \exp Z \times L \mid \\
& \quad B \in \exp Y, \beta, \gamma \in L, \alpha \leqslant \beta * \gamma,(A, B, \beta) \in R,(B, C, \gamma) \in S\}
\end{aligned}
$$

is closed in $\exp X \times \exp Z \times L$. Therefore the set of all subsets of $R \odot S \subset$ $\exp X \times \exp Y \times L$ of the form $\{A\} \times\{B\} \times M$, with $M \underset{\mathrm{cl}}{\subset} L, M \neq \varnothing$, is closed in $\exp (\exp X \times \exp Y \times L)$, hence its image under the continuous correspondence that takes each $\{A\} \times\{B\} \times M$ to $(A, B, \sup M)$ is closed. This image is equal to $R \odot S$. Other properties are obvious. 
Hence we obtain the category of compacta and strict L-ambiguous representations $\mathcal{C S} \mathcal{A m b}_{L}^{*}$. The composition of sequential arrows $R: X \rightarrow Y$ and $S: Y \rightarrow Z$ in this category, i.e. of $R \in \mathcal{C} \mathcal{S} \mathcal{A m b}_{L}(X, Y), S \in \mathcal{C} \mathcal{S A m b}_{L}(Y, Z)$, is equal to $R \underset{*}{\odot} S$. For a compactum $X$, the identity morphism in this category is equal to

$$
\mathbf{1}_{X}=\{(A, B, \alpha) \in \exp X \times \exp X \mid A \subset B \text { or } \alpha=0\} .
$$

If $*$ is not continuous or $L$-ambiguous representations are not strict, then their composition is not necessarily an $L$-ambiguous representation. Therefore we must repeat the trick which was used for the crisp case. For $L$-ambiguous representations $R \subset \exp X \times \exp Y \times L, S \subset \exp Y \times \exp Z \times L$, let $A(R$ ఠ

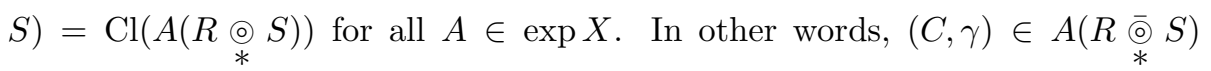
if and only if for all $\gamma^{\prime} \ll \gamma$ and each closed neighborhood $V \supset C$ there are $B_{1}, \ldots, B_{n} \in \exp Y, \alpha_{1}, \beta_{1}, \ldots, \alpha_{n}, \beta_{n} \in L$ such that

$$
\begin{gathered}
\left(A, B_{1}, \alpha_{1}\right), \ldots,\left(A, B_{n}, \alpha_{n}\right) \in R,\left(B_{1}, V, \beta_{1}\right), \ldots,\left(B_{n}, V, \beta_{n}\right) \in S, \\
\alpha_{1} * \beta_{1} \vee \cdots \vee \alpha_{n} * \beta_{n} \geqslant \gamma^{\prime} .
\end{gathered}
$$

If $*=\wedge$, then we write $\bar{\odot}$ for $\underset{*}{\odot}$. If only strict $L$-ambiguous representations are taken, then $\underset{*}{\stackrel{\odot}{*}}=\underset{*}{\odot}$.

Although $\underset{*}{\odot}$ is associative, the composition $\underset{*}{\odot}$ of $L$-ambiguous representations is not associative in the general case. Thus we must impose further restrictions on the class of allowed relations.

For a relation $R \subset \exp X \times \exp Y \times L$ such that all its $\alpha$-cuts are ambiguous representations, we define a relation $R^{\smile} \subset \exp Y \times \exp X \times L$ by the equality $\left(R^{\smile}\right)_{\alpha}=\bigcap_{\beta \ll \alpha}\left(R_{\beta}\right)^{\smile}$. In other words, $(B, A, \alpha) \in R^{\smile}$ if and only if the set $A$ has non-empty intersections with all $A^{\prime} \in \exp X$ such that $B$ is $R_{\beta}$-unavoidable for $A^{\prime}$ for some $\beta \ll \alpha$.

Proposition 3.7. If $R \subset \exp X \times \exp Y \times L$ is an L-ambiguous representation, then so is $R$.

Proof. It is obvious that $R^{\smile} \subset \exp Y \times \exp X \times L$ is closed, contains $\exp Y \times$ $\exp X \times\{0\} \cup \exp Y \times\{X\} \times L$, and $(B, A, \alpha) \in R^{\smile}, B \supset B^{\prime} \in \exp Y, A \subset A^{\prime} \in$ $\exp X, \alpha \geqslant \alpha \in L$ implies $\left(B^{\prime}, A^{\prime}, \alpha^{\prime}\right) \in R^{\smile}$.

Let $(A, B, \alpha),(A, B, \beta) \in R^{\smile}$, then $A \cap A^{\prime} \neq \varnothing$ for all $A^{\prime} \in \exp X$ such that $B \in\left(A^{\prime} R_{\alpha^{\prime}}\right)^{\perp}$ for some $\alpha^{\prime} \ll \alpha$ or $B \in\left(A^{\prime} R_{\beta^{\prime}}\right)^{\perp}$ for some $\beta^{\prime} \ll \beta$. Let $\gamma \ll \alpha \vee \beta$, then due to Lemma 1.1 there are $\alpha^{\prime} \ll \alpha, \beta^{\prime} \ll \beta$ such that $\alpha^{\prime} \vee \beta^{\prime} \geqslant \gamma$. Then

$$
A^{\prime} R_{\gamma} \supset A^{\prime} R_{\alpha^{\prime} \vee \beta^{\prime}}=A^{\prime} R_{\alpha^{\prime}} \cap A^{\prime} R_{\beta^{\prime}} .
$$

Hence

$$
A^{\prime} R_{\gamma}^{\perp} \subset\left(A^{\prime} R_{\alpha^{\prime}} \cap A^{\prime} R_{\beta^{\prime}}\right)^{\perp}=\left(A^{\prime} R_{\alpha^{\prime}}\right)^{\perp} \cup\left(A^{\prime} R_{\beta^{\prime}}\right)^{\perp},
$$


therefore $B \in A^{\prime} R_{\gamma}^{\perp}$ implies $A^{\prime} \cap A \neq \varnothing$, i.e. $(A, B, \alpha \vee \beta) \in R^{\smile}$. Thus $R^{\smile}$ is an $L$-ambiguous representation.

Lemma 3.8. For L-ambiguous representations $R \subset \exp X \times \exp Y \times L, S \subset$

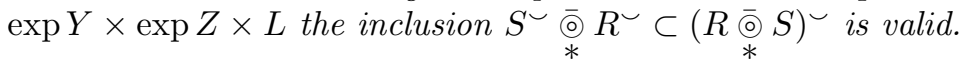

Proof. Let $(C, A, \gamma) \in\left(S^{\smile}\right) \underset{*}{\overline{0}}\left(R^{\smile}\right)$, then for all closed neighborhoods $V \supset A$ and all $\gamma^{\prime} \ll \gamma$ there are $B_{1}, \ldots, B_{n} \in \exp Y, \alpha_{1}, \beta_{1}, \ldots, \alpha_{n}, \beta_{n} \in L$ such that

$$
\begin{gathered}
\left(C, B_{1}, \alpha_{1}\right), \ldots,\left(C, B_{n}, \alpha_{n}\right) \in S^{\smile},\left(B_{1}, V, \beta_{1}\right), \ldots,\left(B_{n}, V, \beta_{n}\right) \in R^{\smile}, \\
\alpha_{1} * \beta_{1} \vee \cdots \vee \alpha_{n} * \beta_{n} \geqslant \gamma^{\prime} .
\end{gathered}
$$

Given an element $\delta \in L$ such that $\delta \ll \gamma$, we choose $\gamma^{\prime} \in L$ such that $\delta \ll \gamma^{\prime} \ll \gamma$.

For all $A^{\prime} \in \exp X$ such that $A^{\prime} \cap V=\varnothing$, and all $i \in\{1, \ldots, n\}, \beta_{i}^{\prime} \ll \beta_{i}$, there is $B_{i}^{\prime} \in \exp Y$ such that $B_{i}^{\prime} \cap B_{i}=\varnothing,\left(A^{\prime}, B_{i}^{\prime}, \beta_{i}^{\prime}\right) \in R$. Similarly, for all $i \in\{1, \ldots, n\}, \alpha_{i}^{\prime} \ll \alpha_{i}$, there is $C_{i}^{\prime} \in \exp Y$ such that $C_{i}^{\prime} \cap C=\varnothing,\left(B_{i}^{\prime}, C_{i}^{\prime}, \alpha_{i}^{\prime}\right) \in$ $S$. Due to the continuity of $\vee$ and the lower semicontinuity of $*$, we can choose $\alpha_{i}^{\prime}, \beta_{i}^{\prime}$ so that

$$
\alpha_{1}^{\prime} * \beta_{1}^{\prime} \vee \cdots \vee \alpha_{n}^{\prime} * \beta_{n}^{\prime} \geqslant \delta .
$$

Then the set $C^{\prime}=C_{1}^{\prime} \cup \cdots \cup C_{n}^{\prime}$ is closed and nonempty, and $C^{\prime} \cap C=\varnothing$,

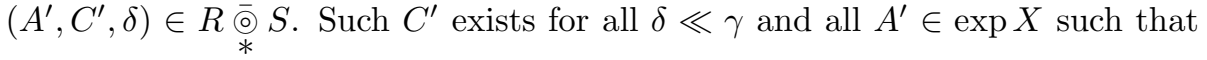
$A^{\prime} \cap V=\varnothing$ for some closed neighborhood $V \supset A$, i.e. for all $A^{\prime} \in \exp X$ such that $A^{\prime} \cap A=\varnothing$. Thus $(C, A, \gamma) \in(\underset{*}{\underset{\odot}{\sigma} S})^{\smile}$.

Proposition 3.9. For an L-ambiguous representation $R \subset \exp X \times \exp Y \times$ $L$, the inclusion $\left(R^{\smile}\right)^{\smile} \subset R$ is valid, and $\left(R^{\smile}\right)^{\smile}=R$ if and only if for all $(A, B, \alpha) \in R, \beta \in L$ such that $\beta \ll \alpha$, and a closed neighborhood $V \supset B$, there is a closed neighborhood $U \supset A$ such that $(U, V, \beta) \in R$.

Proof. By the definition, for all $B \in \exp Y$ :

$$
\begin{gathered}
B R_{\alpha}^{\smile}=\bigcap_{\beta \ll \alpha}\left\{A \in \exp X \mid B \in\left(A R_{\beta}\right)^{\perp}\right\}^{\perp}= \\
\left(\bigcup_{\beta \ll \alpha}\left\{A \in \exp X \mid B \in\left(A R_{\beta}\right)^{\perp}\right\}\right)^{\perp},
\end{gathered}
$$


hence for all $\tilde{A} \in \exp X$ :

$$
\begin{gathered}
A\left(R^{\smile}\right)_{\alpha}^{\smile}=\left(\bigcup_{\beta \ll \alpha}\left\{B \in \exp X \mid A \in\left(B R_{\beta}^{\smile}\right)^{\perp}\right\}\right)^{\perp}= \\
\left(\bigcup_{\beta \ll \alpha}\left\{B \in \exp X \mid A \in\left(\bigcup_{\gamma \ll \beta}\left\{A^{\prime} \in \exp X \mid B \in\left(A^{\prime} R_{\gamma}\right)^{\perp}\right\}\right)^{\perp \perp}\right\}\right)^{\perp}= \\
\left(\bigcup_{\beta \ll \alpha}\left\{B \in \exp X \mid A \in \operatorname{Cl}\left(\bigcup_{\gamma \ll \beta}\left\{A^{\prime} \in \exp X \mid B \in\left(A^{\prime} R_{\gamma}\right)^{\perp}\right\}\right)\right\}\right)^{\perp}= \\
\left(\bigcup_{\beta \ll \alpha}\{B \in \exp X \mid \text { for all } U \in \exp X, A \subset \operatorname{Int} U\right.
\end{gathered}
$$

there is $\gamma \ll \beta$ such that $\left.\left.\left.\left.B \in\left(U R_{\gamma}\right)^{\perp}\right\}\right)\right\}\right)^{\perp}=$

$\left(\bigcup_{\beta \ll \alpha}\left\{B \in \exp X \mid B \in\left(U R_{\beta}\right)^{\perp}\right.\right.$ for all $\left.\left.\left.\left.U \in \exp X, A \subset \operatorname{Int} U\right\}\right)\right\}\right)^{\perp} \subset A R_{\alpha}$.

The equality $\left(A^{\smile}\right)_{\alpha}^{\smile}=A R_{\alpha}$ is equivalent to:

$$
\left.\left.\bigcup_{\beta \ll \alpha}\left\{B \in \exp X \mid B \in\left(U R_{\beta}\right)^{\perp} \text { for all } U \in \exp X, A \subset \operatorname{Int} U\right\}\right)\right\}=\left(A R_{\alpha}\right)^{\perp} .
$$

It fails if and only if there is $\tilde{B} \in \exp Y$ such that $\tilde{B} \in\left(U R_{\beta}\right)^{\perp}$ for all $\beta \ll \alpha, U \in \exp X, A \subset \operatorname{Int} U$, but $\tilde{B} \notin\left(A R_{\alpha}\right)^{\perp}$, i.e. there is $B \in \exp Y$ such that $B \cap \tilde{B}=\varnothing,(A, B, \alpha) \in R$. In this case let $V$ be a closed neighborhood of $B$ such that $V \cap \tilde{B}=\varnothing$, then $V \notin U R_{\beta}$ for all $\beta \ll \alpha$ and closed neighborhoods $U$ of $A$. Thus the condition of the proposition is sufficient for the equality $\left(R^{\smile}\right)^{\smile}=R$. On the contrary, let such $A, B, V$ and $\alpha$ exist, then $\tilde{B}=Y \backslash \operatorname{Int} V$ is a required counterexample, and the condition is necessary.

Definition 3.10. If $R \subset \exp X \times \exp Y \times L$ is an $L$-ambiguous representation such that $\left(R^{\smile}\right)^{\smile}=R$, then we call $R^{\smile}$ pseudo-inverse to $R$, and $R$ is called pseudo-invertible.

We denote the set of pseudo-invertible $L$-ambiguous representations from $X$ to $Y$ by $\mathcal{C P} \mathcal{A m b}_{L}(X, Y)$.

By Lemma 3.8 similarly to Proposition 2.14 we obtain:

Proposition 3.11. Let $R \subset \exp X \times \exp Y \times L$ and $S \subset \exp Y \times \exp Z \times L$ be

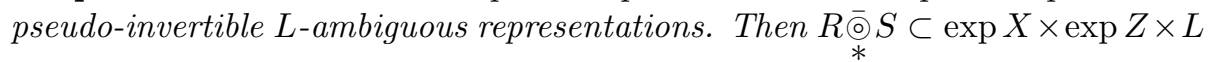

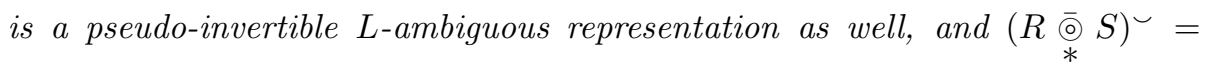
$S^{\sim} \underset{\odot}{\stackrel{\odot}{*}} R^{\smile}$.

Proposition 3.12. For compacta $X, Y$ and a compact Lawson lattice $L$, a relation $R \subset \exp X \times \exp Y \times L$ is an ambiguous representation if and only if for all $A \in \exp X$ the set $A R \subset \exp Y \times L$ is the subgraph of an L-capacity 
$c_{A R} \in M_{L} Y$, and the correspondence $A \mapsto c_{A R}$ is an antitone mapping from $\exp X$ to $M_{L} Y$. This mapping is:

- upper semicontinuous if and only if $R$ is strict;

- lower semicontinuous if and only if $R$ is pseudo-invertible;

- continuous if and only if $R$ is pseudo-invertible and strict.

Definition 3.13. An $L$-ambiguous representation $R$ is called open if both $R$ and $R^{\smile}$ are strict $L$-ambiguous representations, and $\left(R^{\smile}\right)^{\smile}=R$.

Proposition 3.14. A pseudo-invertible strict $L$-ambiguous representation $R \subset$ $\exp X \times \exp Y \times L$ is open if and only if any of the following statements is valid:

- for any open $U \subset X$ and all $\alpha \in L$ the set of $R_{\beta}$-unavoidable sets of all $A \subset U, A \in \exp X$, for all $\beta \in L, \beta \ll \alpha$, is open in the Vietoris topology on $\exp Y$;

- for all $A \in \exp X, A \subset U \underset{\text { op }}{\subset} X, B \in \exp Y$ and $\alpha, \beta \in L$ such that $B \in\left(A R_{\beta}\right)^{\perp}, \beta \ll \alpha$, there are open sets $V_{1}, \ldots, V_{n} \subset Y, \gamma \in L$ and a closed neighborhood $G \supset A$ in $X$ such that $G \subset U, \gamma \ll \alpha, V_{i} \cap B \neq \varnothing, i=1, \ldots, n$, and each $B^{\prime} \in G R_{\gamma}$ contains at least one $V_{i}$.

Proof. To prove that the first statement is equivalent to $R$ being open, it is sufficient to observe that for each $\tilde{A} \in \exp X$ the complement $\exp Y \backslash R \smile \tilde{A}$ is equal to the set of $R_{\beta}$-unavoidable sets for all $A \in \exp X, A \subset U=X \backslash \tilde{A}$, $\beta \ll \alpha$.

Let $R$ be an open $L$-ambiguous representation, hence $R^{\smile}$ is a closed subset, and let $A \in \exp X$ be such that $B \in\left(A R_{\beta}\right)^{\perp}$ for some $\beta \ll \alpha$. If we take $A_{0}=X \backslash U$, then $\left(A_{0}, B, \alpha\right) \notin R^{\smile}$, hence there must exist neighborhoods $U^{\prime} \supset A_{0}$ in $X,\left\langle V_{1}, \ldots, V_{n}\right\rangle \ni B$ in $\exp Y, W_{\alpha} \ni \alpha$ in $L$ such that, for all $A^{\prime} \in \exp X, A^{\prime} \subset U^{\prime}, B^{\prime} \in\left\langle V_{1}, \ldots, V_{n}\right\rangle, \alpha^{\prime} \in W_{\alpha}$, there is $A^{\prime \prime} \in \exp X$ such that $A^{\prime} \cap A^{\prime \prime}=\varnothing$ and $B^{\prime}$ is $R_{\beta^{\prime}}$-unavoidable for $A^{\prime \prime}$ for some $\beta^{\prime} \ll \alpha^{\prime}$. If necessary, we can make $V_{i}$ smaller to be disjoint. We can also choose $U^{\prime}$ so that $\mathrm{Cl} U^{\prime} \cap A=\varnothing$. Moreover, $B^{\prime}$ is $R_{\beta^{\prime}}$-unavoidable for $G=X \backslash U^{\prime}$, and $G$ is a closed neighborhood of $A$. The lattice $L$ is compact Lawson, hence there is an open neighborhood $O_{\alpha} \ni \alpha, O_{\alpha} \subset W_{\alpha}$, such that inf $O_{\alpha} \in W_{\alpha}$. Let $\gamma=\inf O_{\alpha}$, then $\gamma \ll \alpha$, and each $B^{\prime} \in\left\langle V_{1}, \ldots, V_{n}\right\rangle$ is $R_{\gamma}$-unavoidable for $G$. It is possible if and only if each element of $G R_{\gamma}$ is a superset of some of $V_{i}$. Necessity of the second statement is proved.

Now the proof of its sufficiency is obvious.

Proposition 3.15. Let $*: L \times L \rightarrow L$ be open, $R \subset \exp X \times \exp Y \times L$ and $S \subset \exp Y \times \exp Z \times L$ open L-ambiguous representations. Then $R \odot S \subset$ $\exp X \times \exp Z \times L$ is an open L-ambiguous representation as well.

Proof. By Proposition 3.11, the relation $(R \underset{*}{\odot} S)^{\smile}$ is equal to the composition of two strict $L$-ambiguous representations $S^{\smile}$ and $R^{\smile}$, hence is a strict $L$ ambiguous representation itself. 
By Proposition 3.9 the composition of pseudo-invertible $L$-ambiguous representations is associative. Thus we obtain a collection of the categories of compacta and pseudo-invertible $L$-ambiguous representations $\mathcal{C P} \mathcal{A} \mathrm{mb}_{L}^{*}$ with the same objects (=compacta) and morphisms (=pseudo-invertible $L$-ambiguous representations), but with different laws of composition $\underset{*}{\underset{\sim}{\rightleftharpoons}}$ parameterized by certain t-norms on the lattice $L$. For all of them there is an involutive isomorphism $\left.(-)^{\smile}:(\mathcal{C P} \mathcal{A m b})^{*}\right)^{o p} \rightarrow \mathcal{C P} \mathcal{A} \mathrm{mb}_{L}^{*}$ which preserves objects. For $*$ continuous, each of these categories contains a subcategory $\mathcal{C O} \mathcal{A m b}{ }_{L}^{*}$ with all open $L$-ambiguous representations as morphisms and the composition law $\underset{*}{\stackrel{0}{\odot}}=\underset{*}{\odot}$.

We also denote the intersection of $\mathcal{C P} \mathcal{A m b}{ }_{L}^{*}$ and $\mathcal{C} \mathcal{S} \mathcal{A m b}{ }_{L}^{*}$ by $\mathcal{C P S} \mathcal{A} \mathrm{mb}_{L}^{*}$. The identity arrows for these categories are the same as in $\mathcal{C S} \mathcal{A m b}{ }_{L}^{*}$.

Again, if $*=\wedge$, we omit it in the notation for categories.

Each ambiguous representation $R \subset \exp X \times \exp Y$ can be identified with an $L$-ambiguous representation $R_{L}$ defined as follows:

$$
R_{L}=\{(A, B, \alpha) \in \exp X \times \exp Y \mid(A, B) \in R \text { or } \alpha=0\} .
$$

Then the categories of $\mathcal{C} \mathcal{S} \mathcal{A m b}, \mathcal{C P} \mathcal{A m b}, \mathcal{C P S} \mathcal{A m b}$, and $\mathcal{C O} \mathcal{A m b}$ are embedded into the respective categories of $\mathcal{C} \mathcal{S} \mathcal{A m b}{ }_{L}^{*}, \mathcal{C P} \mathcal{A m b}{ }_{L}^{*}, \mathcal{C P S} \mathcal{A m b}{ }_{L}^{*}$, and $\mathcal{C O} \mathcal{A m b}{ }_{L}^{*}$ (independently of $*$ ).

When we attempt to study order and topological properties of sets of (strict, open) $L$-ambiguous representations in the same manner as we did before for (non-fuzzy) representations, we encounter new difficulties. If $\mathcal{C} \mathcal{A m b} b_{L}(X, Y)$ is ordered by inclusion, then the top and the bottom elements of this poset are obvious:

$\top_{X, Y, L}=\exp X \times \exp Y \times L, \perp_{X, Y, L}=\exp X \times\{Y\} \times L \cup \exp X \times \exp Y \times\{0\}$.

If $R, S \in \mathcal{C} \mathcal{A m b}_{L}(X, Y)$, then $R \cap S \in \mathcal{C} \mathcal{A m b}(X, Y)$, but, for $|X|>1,|Y|>1$ and a non-linearly ordered $L$, not always $R \cup S \in \mathcal{C} \mathcal{A} \operatorname{Abb}_{L}(X, Y)$. E.g. let $\alpha, \beta \in L$ be incomparable, $x_{1}, x_{2} \in X, y \in Y$ and

$$
\begin{aligned}
& R=\perp_{X, Y, L} \cup\left\{\left(\left\{x_{1}\right\}, F, \gamma\right) \mid y \in F \in \exp Y, \gamma \in L, 0 \leqslant \gamma \leqslant \alpha\right\}, \\
& S=\perp_{X, Y, L} \cup\left\{\left(\left\{x_{2}\right\}, F, \gamma\right) \mid y \in F \in \exp Y, \gamma \in L, 0 \leqslant \gamma \leqslant \beta\right\},
\end{aligned}
$$

then $R$ and $S$ are $L$-ambiguous representations, but, if $x_{1}=x_{2}$, then $R \cup S$ is not. This implies that, for an infinite $X,|Y|>1$, and a non-linearly ordered $L$, the set $\mathcal{C} \mathcal{S} \mathcal{A m b}_{L}(X, Y)$ is not closed in $\exp (\exp X \times \exp Y \times L)$, although its elements are closed sets.

It is easy to describe suprema and infima in $\mathcal{C} \mathcal{A m b}_{L}(X, Y)$ and $\mathcal{C} \mathcal{S} \mathcal{A m b}_{L}(X, Y)$. For a set $\mathcal{R} \subset \mathcal{C} \mathcal{A m b}_{L}(X, Y)$ its lowest upper bound is a relation $R_{0} \subset \exp X \times$ $\exp Y \times L$ defined by the equality

$$
\begin{aligned}
A R_{0}= & \operatorname{Cl}\{(B, \gamma) \in \exp Y \times L \mid \gamma \leqslant \sup \{\alpha \in L \mid \\
& (A, B, \alpha) \in R \text { for some } R \in \mathcal{R}\}\}
\end{aligned}
$$


for all $A \in \exp X$.

Similarly, if a strict $L$-ambiguous representation $S$ is an upper bound of a subset $\mathcal{R}$ in $\mathcal{C} \mathcal{S} \mathcal{A m b}_{L}(X, Y)$, then $S$ must contain a set

$$
\begin{gathered}
R_{0}=\operatorname{Cl}\{(A, B, \gamma) \in \exp X \times \exp Y \times L \mid \gamma \leqslant \sup \{\alpha \in L \mid \\
(A, B, \alpha) \in R \text { for some } R \in \mathcal{R}\}\} .
\end{gathered}
$$

It is obvious that $R_{0}$ is a strict $L$-ambiguous representation and a least upper bound of $\mathcal{R}$.

The greatest lower bound of a subset $\mathcal{R}$ of $\mathcal{C} \mathcal{A m b}{ }_{L}(X, Y)$ or $\mathcal{C} \mathcal{S} \mathcal{A m b}_{L}(X, Y)$ is simply the intersection of $\mathcal{R}$.

In both these sets the pairwise supremum of $R_{1}, R_{2}$ is determined by the equality

$$
R_{1} \vee R_{2}=\left\{\left(A, B, \alpha_{1} \vee \alpha_{2}\right) \mid\left(A, B, \alpha_{1}\right) \in R_{1},\left(A, B, \alpha_{2}\right) \in R_{2}\right\} .
$$

Proposition 3.16. Let $R, S \in \mathcal{C} \mathcal{A} \operatorname{mb}_{L}(X, Y)$, then $(R \vee S)^{\smile}=R^{\smile} \vee S^{\smile}$, $(R \wedge S)^{\smile}=R^{\smile} \wedge S^{\smile}$.

Proof. The operation $(-)^{\smile}$ is isotone, hence $(R \vee S)^{\smile} \supset R^{\smile} \vee S^{\smile},(R \wedge S)^{\smile} \subset$ $R^{\smile} \wedge S^{\smile}$.

Let $(B, A, \gamma) \notin R^{\smile} \vee S^{\smile}$, i.e. for all $\alpha, \beta \in L$ such that $\alpha \vee \beta \geqslant \gamma$, we have either $(B, A, \alpha) \notin R^{\smile}$ or $(B, A, \beta) \notin S^{\smile}$, i.e. either there are $A^{\prime} \in \exp X$, $\alpha^{\prime} \ll \alpha$ such that $A^{\prime} \cap A=\varnothing, B \in A^{\prime} R_{\alpha^{\prime}}$, or there are $A^{\prime} \in \exp X, \beta^{\prime} \ll \beta$ such that $A^{\prime} \cap A=\varnothing, B \in A^{\prime} S_{\beta^{\prime}}$. The set $\left\{(\alpha, \beta) \in L^{2} \mid \alpha \vee \beta \geqslant \gamma\right\}$ is compact, therefore there is a finite collection $\alpha_{1}, \ldots, \alpha_{m}, \beta_{1}, \ldots, \beta_{n} \in L$ and a closed nonempty set $A^{\prime} \subset X$ such that $A^{\prime} \cap A=\varnothing$,

$$
B \in\left(A^{\prime} R_{\alpha_{1}}\right)^{\perp} \cap \cdots \cap\left(A^{\prime} R_{\alpha_{m}}\right)^{\perp} \cap\left(A^{\prime} S_{\beta_{1}}\right)^{\perp} \cap \cdots \cap\left(A^{\prime} S_{\beta_{n}}\right)^{\perp},
$$

and, for all $\alpha, \beta \in L$ such that $\alpha \vee \beta \geqslant \gamma$, either $\alpha_{i} \ll \alpha$ for some $1 \leqslant i \leqslant m$, or $\beta_{j} \ll \beta$ for some $1 \leqslant j \leqslant n$. Hence there is $\gamma^{\prime} \ll \gamma$ such that, for all $\alpha, \beta \in L$ such that $\alpha \vee \beta \geqslant \gamma^{\prime}$, either $\alpha_{i} \ll \alpha$ for some $1 \leqslant i \leqslant m$, or $\beta_{j} \ll \beta$ for some $1 \leqslant j \leqslant n$. Then $(R \vee S)_{\gamma^{\prime}} \subset R_{\alpha_{1}} \cup \cdots \cup R_{\alpha_{m}} \cup S_{\beta_{1}} \cup \cdots \cup S_{\beta_{m}}$, and

$$
\left(A^{\prime}(R \vee S)_{\gamma^{\prime}}\right)^{\perp} \supset\left(A^{\prime} R_{\alpha_{1}}\right)^{\perp} \cap \cdots \cap\left(A^{\prime} R_{\alpha_{m}}\right)^{\perp} \cap\left(A^{\prime} S_{\beta_{1}}\right)^{\perp} \cap \cdots \cap\left(A^{\prime} S_{\beta_{n}}\right)^{\perp},
$$

therefore $B \in\left(A^{\prime}(R \vee S)_{\gamma^{\prime}}\right)^{\perp}$ for some $\gamma^{\prime} \ll \gamma, A^{\prime} \in \exp X, A^{\prime} \cap A=\varnothing$, thus $(B, A, \gamma) \notin(R \vee S)^{\smile}$. We have proved that $R^{\smile} \vee S^{\smile}=(R \vee S)^{\smile}$.

Let $(B, A, \gamma) \in R^{\smile} \wedge S^{\smile}$, then for all $\gamma^{\prime} \ll \gamma$ and $A^{\prime} \in \exp X, A^{\prime} \cap A=\varnothing$, there are $B_{1} \in A^{\prime} R_{\gamma^{\prime}}$ and $B_{2} \in A^{\prime} S_{\gamma^{\prime}}$ such that $B_{1} \cap B=B_{2} \cap B=\varnothing$. Then $B^{\prime}=B_{1} \cup B_{2} \in A^{\prime}(R \wedge S)_{\gamma^{\prime}}, B^{\prime} \cap B=\varnothing$, hence $(B, A, \gamma) \in(R \wedge S)^{\smile}$. The equality $R^{\smile} \wedge S^{\smile}=(R \wedge S)^{\smile}$ is also proved.

Corollary 3.17. If $R, S$ are elements of $\mathcal{C P} \mathcal{A m b}_{L}(X, Y)\left(\operatorname{or}_{\mathcal{C P S}} \mathcal{A m b}_{L}(X, Y)\right.$, or $\mathcal{C O} \mathcal{A m b}_{L}(X, Y)$ ), then $R \vee S$ and $R \wedge S$ are also in $\mathcal{C P} \mathcal{A m b}_{L}(X, Y)$ (resp. in $\mathcal{C P S} \mathcal{A m b}_{L}(X, Y)$ or $\left.\mathcal{C O} \mathcal{A m b}{ }_{L}(X, Y)\right)$. 
Thus $\mathcal{C P} \mathcal{A m b}_{L}(X, Y), \mathcal{C P S} \mathcal{A m b}_{L}(X, Y)$ and $\mathcal{C O} \mathcal{A m b}_{L}(X, Y)$ are sublattices of the lattice $\mathcal{C} \mathcal{A m b} \operatorname{mb}_{L}(X, Y)$.

To address topological issues, we define for each $R \in \mathcal{C} \mathcal{S} \mathcal{A m b}_{L}(X, Y)$ a relation $R^{\cup} \subset \exp ^{2} X \times \exp Y \times L$ by the equality

$$
\begin{gathered}
R^{\cup}=\left\{(\mathcal{A}, B, \gamma) \mid \mathcal{A} \in \exp ^{2} X, B \in \exp Y, \gamma \in L,\right. \\
\gamma \leqslant \sup \{\alpha \in L \mid(A, B, \alpha) \in R \text { for some } A \in \mathcal{A}\}\} .
\end{gathered}
$$

Observe that for $A \in \exp X, B \in \exp Y, \alpha \in L$ and $R \in \mathcal{C} \mathcal{S} \mathcal{A m b}_{L}(X, Y)$ the inclusions $(A, B, \alpha) \in R$ and $(\{A\}, B, \alpha) \in R^{\cup}$ are equivalent, therefore the correspondence $R \mapsto R^{\cup}$ is injective. Let us consider the image of this correspondence.

Proposition 3.18. The relations $R^{\cup}$ for all $R \in \mathcal{C} \mathcal{S} \mathcal{A m b}_{L}(X, Y)$ are closed sets and form a closed subset of $\exp \left(\exp ^{2} X \times \exp Y \times L\right)$.

In order to prove this proposition, we define an operation on $\exp \left(\exp ^{2} X \times\right.$ $\exp Y \times L)$ such that all sets of the form $R^{\cup}$ are fixed points of this operation.

For a compactum $X$ and a closed non-empty set $\mathcal{A} \subset \exp X$, the set

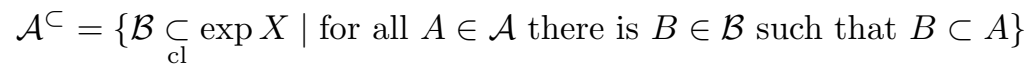

is an inclusion hyperspace, i.e. an element of $G(\exp X) \subset \exp ^{3} X$.

Lemma 3.19. The correspondence $\mathcal{A} \mapsto \mathcal{A}^{\complement}$ is a continuous mapping $\exp ^{2} X \rightarrow$ $G(\exp X)$.

Proof. Let $W$ be an open subset of $\exp X$, then the preimages of subbase elements of $G(\exp X)$

$$
\begin{gathered}
\left\{\mathcal{A} \in \exp ^{2} X \mid \mathcal{A}^{\subset} \in W^{+}\right\}=\left\{\mathcal{A} \in \exp ^{2} X \mid \text { for all } A \in \mathcal{A} \text { there is } B \in W\right. \\
\text { such that } B \subset A\}=\langle W \uparrow\rangle,
\end{gathered}
$$

and

$\left\{\mathcal{A} \in \exp ^{2} X \mid \mathcal{A}^{\complement} \in W^{-}\right\}=\left\{\mathcal{A} \in \exp ^{2} X \mid\right.$ there is $A \in \mathcal{A}$ such that $B \in W$

for all $B \in \exp X$ such that $B \subset A \in \mathcal{A}\}=\langle\exp X, \exp X \backslash(\exp X \backslash W) \uparrow\rangle$

are open, thus the mapping in question is continuous.

Corollary 3.20. For a closed relation $T \subset \exp ^{2} X \times \exp Y \times L$ the relation

$$
T^{\subset}=\bigcup\left\{\mathcal{A}^{\subset} \times\{B\} \times\{\alpha\} \downarrow \mid(\mathcal{A}, B, \alpha) \in T\right\}
$$

is closed, continuously depends on $T$, and $\left(T^{\subset}\right)^{\subset}=T^{\subset} \supset T$.

For a closed relation $T \subset \exp ^{2} X \times \exp Y \times L$ we define a relation $T^{\text {sup }} \subset$ $\exp ^{2} X \times \exp Y \times L$ by the equality:

$$
T^{\text {sup }}=\left\{\left(\bigcup \exp \operatorname{pr}_{1}(\mathcal{F}), \bigcup \exp \operatorname{pr}_{2}(\mathcal{F}), \sup \exp \operatorname{pr}_{3}(\mathcal{F})\right) \mid \mathcal{F} \underset{\mathrm{cl}}{\subset} T, \mathcal{F} \neq \varnothing\right\},
$$

where $\operatorname{pr}_{i}, i=1,2,3$, are the projections of the product $\exp (\exp X) \times \exp Y \times L$ onto the respective factors. 
Lemma 3.21. The set $T^{\text {sup }}$ is closed, satisfies $\left(T^{\text {sup }}\right)^{\text {sup }}=T^{\text {sup }} \supset T$, and the mapping that takes each $T$ to $T^{\text {sup }}$ is continuous.

The proof is obvious and uses the fact that for each compactum $K$ the mapping $U: \exp ^{2} K \rightarrow \exp K$ is continuous.

Proof of the proposition. For a strict $L$-ambiguous representation $R \subset \exp X \times$ $\exp Y \times L$ the set $R^{\cup}$ is equal to $\left(R_{\bullet}\right)^{\text {sup }}$, where

$$
R_{\bullet}=\{(\{A\}, B, \alpha) \mid(A, B, \alpha) \in R\} .
$$

The closedness of $R_{\bullet}$ implies that $R^{\cup}$ is closed.

For each closed relation $T \subset \exp ^{2} X \times \exp Y \times L$ we put

$$
T^{+}=\left(\left(T \cup \exp ^{2} X \times\{Y\} \times L \cup \exp ^{2} X \times \exp Y \times\{0\}\right)^{\complement}\right)^{\text {sup }} .
$$

Then $T^{+} \subset \exp ^{2} X \times \exp Y \times L$ is closed, continuously depends on $T$, and $\left(T^{+}\right)^{+}=T^{+} \supset T$. Moreover, $T=R^{\cup}$ for some $R \in \mathcal{C} \mathcal{S} \mathcal{A m b}_{L}(X, Y)$ if and only if

$$
T=T^{+}=(T \cap\{(\{A\}, B, \alpha) \mid A \in \exp X, B \in \exp Y, \alpha \in L\})^{+} .
$$

The latter equality selects a closed subset of $\exp \left(\exp ^{2} X \times \exp Y \times L\right)$.

Therefore we define a compact Hausdorff topology on the set $\mathcal{C} \mathcal{S} \mathcal{A m b}_{L}(X, Y)$ by the requirement that the mapping that takes each $R \in \mathcal{C} \mathcal{S} \mathcal{A m b}_{L}(X, Y)$ to $R^{\cup} \in \exp \left(\exp ^{2} X \times \exp Y \times L\right)$ is an embedding.

For all $R, S \in \mathcal{C} \mathcal{S} \operatorname{Amb}_{L}(X, Y)$ the inclusions $R \subset S$ and $R^{\cup} \subset S^{\cup}$ are equivalent. The partial order on $\mathcal{C S} \mathcal{A m b}_{L}(X, Y)$ is closed, hence for $\mathcal{R} \subset$ $\mathcal{C S} \mathcal{A m b}_{L}(X, Y)$ we have $\sup \mathcal{R}=\sup \mathrm{Cl} \mathcal{R}$. Therefore we further assume that $\mathcal{R}$ is closed. Observe that $(\bigcup \mathcal{R}) \bullet=\bigcup\{R \bullet \mid R \in \mathcal{R}\}$. For any upper bound $S$ of $\mathcal{R}$ the relation $S^{\cup}$ should contain

$$
(\bigcup \mathcal{R})^{\cup}=\left(\bigcup\left\{R_{\bullet} \mid R \in \mathcal{R}\right\}\right)^{+}=\left(\bigcup\left\{\left(R_{\bullet}\right)^{+} \mid R \in \mathcal{R}\right\}\right)^{+}=\left(\bigcup\left\{R^{\cup} \mid R \in \mathcal{R}\right\}\right)^{+}
$$

and the latter set satisfies the last equality from the proof of the previous proposition. Therefore the least upper bound of $\mathcal{R}$ is determined by the equality

$$
(\sup \mathcal{R})^{\cup}=\left(\bigcup\left\{R^{\cup} \mid R \in \mathcal{R}\right\}\right)^{+} .
$$

This formula also implies that the mapping that takes each closed set $\mathcal{R}$ to $\sup \mathcal{R}$ is continuous, hence $\mathcal{C S} \mathcal{A m b}_{L}(X, Y)$ is a compact Lawson upper semilattice. The infimum in this lattice is in general not continuous.

Question 3.22. When does $\mathcal{C O} \mathcal{A m b}_{L}(X, Y)$ have top and bottom elements? What are topological properties of the subsets $\mathcal{C P S} \mathcal{A m b}_{L}(X, Y), \mathcal{C O} \mathcal{A m b} \mathrm{m}_{L}(X, Y)$ of $\mathcal{C S} \mathcal{A m b}_{L}(X, Y)$ ? 


\section{Epilogue}

Of course, interpretation of ambiguous representations is itself somewhat ambiguous. We propose only one of possible (known to these authors) possibilities. Let an object be a closed subset $B$ of a compactum $Y$ (an object space). The object is not accessible by us directly, but we apply some procedures (series of procedures) to elements of $B$ (or entire $B$ ) to obtain a closed set $A$ in a compactum $X$ (a representation space) that represents (in some sense) the original set $B$. This information is subject to random and systematic interferences, hence for a fixed $B$ the result is ambiguous, and even disjoint $A$ can be obtained. This information can also be incomplete, therefore, if a set $A$ represents a set $B$, then $A$ can also represent a larger set $B^{\prime} \supset B$. Likewise, if $A$ is obtained as a representation of $B$, then any non-empty closed $A^{\prime} \subset A$ can also be obtained for the same $B$, e.g. if less attempts to obtain information have been made.

It is natural to demand that the relation " $A$ can represent $B$ " be closed (=topologically stable), i.e. if $A$ are valid representations of $B_{i}$, and $B_{i}$ converge to $B_{0}$, then $A$ should also be a valid representation of $B_{0}$. If the same (optionally) is true for representing sets, then the representation is called strict. Thus we obtain a binary relation $R$ between the sets $\exp X$ and $\exp Y$ of nonempty closed subsets of $X$ and $Y$, and strict representations are characterized by the property that they are closed in the product of $\exp X$ and $\exp Y$ with the Vietoris topologies.

A "pseudo-inverse" to $R$ ambiguous representation $R^{\smile} \subset \exp Y \times \exp X$ appears when, given a representing set in $X$, we are interested in areas in $Y$ which are outside of the object. Namely, $(\tilde{B}, \tilde{A}) \in R^{\smile}$ if and only if all closed non-empty $A$ outside of $\tilde{A}$ can represent via $R$ some non-empty closed $B$ outside of $\tilde{B}$. If $\left(R^{\smile}\right)^{\smile}=R$, we call $R$ pseudo-invertible. The relation $R^{\smile}$ is closed only if $R$ satisfies a requirement similar to openness of a mapping. If the equality $\left(R^{\smile}\right)^{\smile}=R$ is also valid, we call such $R$ an open ambiguous representation, and $R^{\smile}$ belongs to the same class.

For ambiguous representations the composition law is defined, which expresses formally an intuitive fact that representations (procedures of obtaining information) can be combined sequentially. Although this law is not associative in general, strict, pseudo-invertible and open representations form respectively categories $\mathcal{C} \mathcal{S} \mathcal{A m b}, \mathcal{C P} \mathcal{A m b}$ and $\mathcal{C O} \mathcal{A m b}$. The operation $(-)^{\smile}$ determines antiisomorphisms from the categories $\mathcal{C P} \mathcal{A m b}$ and $\mathcal{C O} \mathcal{A m b}$ onto themselves.

It can be useful that, for given compacta $X, Y$, the set $\mathcal{C S A m b}(X, Y)$ of strict ambiguous representations between $X$ and $Y$ is a compact Hausdorff space and a complete lattice with respect to inclusion, and open representations form a sublattice $\mathcal{C O} \mathcal{A m b}(X, Y)$. It allows one to compare and approximate representations.

It is natural that a set $A \subset X$ can represent different $B \subset Y$ with different level of acceptability. We propose to express this level as an element of a lattice $L$. An $L$-fuzzification of the above theory is also provided in the paper. To interact well with compacta, $L$ must be a compact Hausdorff Lawson lattice (possess local bases that consist of sublattices). This class includes the most common 
case $L=[0,1]$. Then a strict $L$-ambiguous representation is a closed $L$-relation between $\exp X$ and $\exp Y$, i.e. a closed subset $R \subset \exp X \times \exp Y \times L$, with certain properties. An equivalent, but sometimes more convenient interpretation: for all $A \in \exp X$ we fix an $L$-capacity $c_{A R}$ (cf. [17]). It is a function that sends each $B \in \exp Y$ to an element $c_{A R}(B) \in L$ that shows how appropriate is $A$ as a representation of $B$ (the more, the better). To reflect the fact that successive application of uncertain conclusions can give even more uncertain result, we propose to use generalized triangular norms $*$ on $L$ [1] to define compositions. Thus we obtain a collection of categories $\mathcal{C S} \mathcal{A m b}{ }_{L}^{*}, \mathcal{C P} \mathcal{A} \mathrm{mb}_{L}^{*}, \mathcal{C P S} \mathcal{A m b}{ }_{L}^{*}$, and $\mathcal{C O} \mathcal{A m b}{ }_{L}^{*}$

The definition of fuzzy ambiguous representation allows at least as many interpretations as the definitions of fuzzy set and fuzzy relation. Hence we shall not discuss them here and refer the reader to 2, 3]. Virtually any of semantics of fuzzy sets considered in the latter citations can be meaningfully applied to the objects defined in this paper.

We expect that ambiguous representations will become a convenient framework for problems of image recognition and data mining, allowing to apply methods of topology and category theory. Interplay between this theory, fuzzy

and rough sets/relations, and functors and monads in the category of compacta will be the topic of our next paper.

The authors wish to express gratitude to anonymous reviewers and editors for valuable comments, corrections and suggestions.

\section{References}

[1] C.A. Drossos, Generalized t-norm structures, Fuzzy Sets and Systems 104:1 (1999) 53-59.

[2] D. Dubois, H. Prade, The three semantics of fuzzy sets, Fuzzy Sets and Systems 90 (1997) 141-150.

[3] D. Dubois, H. Prade, Gradualness, uncertainty and bipolarity: Making sense of fuzzy sets, Fuzzy Sets and Systems (2010), doi:10.1016/j.fss.2010.11.007.

[4] W.L. Gau, J. Buehrer, Vague sets, IEEE Transactions on Systems, Man and Cybernetics 23 (1993) 610-614.

[5] M. Gehrke, C. Walker, E. Walker, Some comments on interval based fuzzy sets, Int. J. of Intelligent Sys. 11 (1996) 751-759.

[6] J.A. Goguen, L-fuzzy sets, J. Math. Anal. Appl. 18 (1967) 145-157.

[7] U. Höhle, Fuzzy sets and sheaves. Part I: Basic concepts, Fuzzy Sets and Systems 158:11 (2007) 1143-1174.

[8] U. Höhle, Fuzzy sets and sheaves. Part II: Sheaf-theoretic foundations of fuzzy set theory with applications to algebra and topology, Fuzzy Sets and Systems 158:11 (2007) 1175-1212. 
[9] A. Kehagias, M. Konstantinidou, L-fuzzy valued inclusion measure, L-fuzzy similarity and L-fuzzy distance, Fuzzy Sets and Systems 136:3 (2003) 313332 .

[10] J.D. Lawson, Topological semilattices with small semilattices, J. Lond. Math. Soc. 11 (1969) 719-724.

[11] T.J. Li, Rough approximation operators on two universes of discourse and their fuzzy extensions, Fuzzy Sets and Systems 159 (2008) 3033-3050.

[12] T.Y. Lin, Neighborhood systems: a qualitative theory for fuzzy and rough sets, in: Paul Wang (Ed.), Advances in Machine Intelligence and Soft Computing, Volume IV, 1997, pp. 132-155.

[13] S. Mac Lane, Categories for the Working Mathematician. 2nd ed. Springer, N.Y., 1998.

[14] E. Michael, Topologies on spaces of subsets, Trans. Amer. Math. Soc. 71 (1951) 152-182.

[15] E.V. Moiseev, On spaces of closed growth and inclusion hyperspaces, Vestnik Mosk. Univ., Ser. Math. Mech. 3 (1988) 54-57.

[16] C.V. Negoita, D.A. Ralescu, Representation theorems for fuzzy concepts, Kybernetes 4 (1975) 169-174.

[17] O.R. Nykyforchyn, Capacities with values in compact Hausdorff lattices, Applied Categorical Structures 15:3 (2008) 243-257.

[18] O.R. Nykyforchyn, D. Repovš, Inclusion hyperspaces and capacities on Tychonoff spaces: functors and monads, Topology and Its Appl. 157:15, (2009) 2421-2434.

[19] Z. Pawlak, Rough Sets, Int. J. Computer and Information Sciences 5 (1982) 341-356.

[20] Shostak A.P. Two decades of fuzzy topology: basic ideas, notions and results, Russian Math. Surveys 44:6 (1989) 125-186.

[21] T. Terano, K. Asai, M. Sugeno, Fuzzy Sets Theory and Its Applications, Academic Press, San Diego, 1992.

[22] M. Winter, Goguen Categories: A Categorical Approach to L-fuzzy Relations, 1st edition. Dordrecht, Springer, Trends In Logic 25, 2007.

[23] L.A. Zadeh, Fuzzy sets, Information and Control 8 (1965) 338-353.

[24] L.A. Zadeh, The concept of a linguistic variable and its application to approximate reasoning, Inform. Sci. 8 (1975) 199-249. 